\title{
Targeted Advertising on Competing Platforms
}

\author{
Qiang Gong, Siqi Pan, and Huanxing Yang*
}

November 2017

\begin{abstract}
This paper studies targeted advertising in two-sided markets. Two platforms, with different targeting abilities, compete for single-homing consumers, while advertising firms are multi-homing. Ads overall impose negative externalities on consumers. When the targeting ability of the advantaged platform increases, (i) the advantaged platform will have more advertising firms, attract more consumers, and become more profitable, but its ad price and total volume of ads could either increase or decrease; (ii) the disadvantaged platform will have fewer advertising firms, attract fewer consumers, have fewer ads in total, increase its ad price, and become less profitable; (iii) all consumers will be better off. Finally, we compare social incentives and equilibrium incentives in investing in targeting ability, and find that underinvestment is most likely to occur.
\end{abstract}

Key Words: Targeting; Advertising; Two-sided markets; Online Platforms

JEL Classification: D43, L13, L15

\section{Introduction}

It is well known that online advertising is endowed with a higher targeting ability than offline advertising. This is mainly because online platforms are able to track consumers' web browsing activities, thus sending them ads more closely related to their interests. The high targeting ability of online advertising brings two potential benefits. For firms, it can make advertising more effective as their ads are more likely to reach a relevant consumer. For consumers, it can reduce the nuisance caused by irrelevant ads as they are more likely to view ads that fit their interests. Probably for these reasons, the aggregate spending of advertising on the Internet has increased dramatically in recent years, while that of traditional media, including TV, newspaper, and radio, has declined steadily.

\footnotetext{
${ }^{*}$ Qiang Gong: Wenlan School of Economics, Zhongnan University of Economics and Law, China; qianggong@zuel.edu.cn. Siqi Pan: Department of Economics, University of Melbourne, Australia; siqi.pan@unimelb.edu.au. Huanxing Yang: Department of Economics, Ohio State University, USA; yang.1041@osu.edu.

${ }^{\dagger}$ We would like to thank the Editor, two anonymous referees, Yongmin Chen, the audience of the 12th Annual IIOC (Chicago, April 2014), and the seminar audience of Zhejiang University, for helpful comments.
} 
Under the framework of two-sided markets, this paper studies how differential targeting technologies of competing platforms affect the advertising market. In our model, consumers/viewers and advertising firms are brought together by two competing platforms with different targeting abilities; ads overall impose negative externalities on consumers. Such a setting allows us to ask the following research questions. How do differential targeting abilities and changes in targeting ability affect the prices of ads, the number of advertising firms, and the total volume of ads? Do consumers always benefit from increases in targeting ability? Do increases in targeting ability have different impacts on different types of firms? Compared to the social optimum, do platforms invest too little or too much in improving their targeting technologies?

One important feature of the environment studied in this paper is that platforms are playing an active role in identifying relevant consumers and setting prices. Not only does such a feature captures the common practice in many real markets, but it also distinguishes our model from the previous literature and generates different predictions. Targeted advertising has received a considerable amount of attention in the literature. While most of the earlier papers (see the references later) study how targeted advertising affects competition in product markets, several recent papers (Athey and Gans, 2010; Bergemann and Bonatti, 2011; and Johnson, 2013) have shifted their focus to the advertising market. In Johnson (2013), consumers and advertising firms are brought together for potential match; consumers incur nuisance costs when viewing irrelevant ads. With a single (implicit) platform, the paper investigates the impacts of increasing targeting ability on market outcomes. Since the platform is not strategically active, the advertising price in Johnson (2013) is exogenously fixed. In contrast, the prices in our model are endogenously set by platforms, which, as we will explain shortly, generates different predictions especially on consumer welfare.

Athey and Gans (2010, AG henceforth) and Bergemann and Bonatti (2011, BB henceforth) study the impact of targeting on the allocation and the price of ads. In AG, a general media outlet competes with many local media outlets. They identify a supply-side impact of targeting (at the general outlet): it allows a more efficient allocation of scarce advertising space, and the resulting increase in space supply may push down ad price. In BB's model, there is a continuum of advertising markets; every market is perfectly competitive. One of the markets is large or general while the rest are specialized. When the targeting ability of ads increases, it always occurs in every specialized market. ${ }^{1}$ Again, due to the perfectly competitive environment, the platforms in $\mathrm{BB}$ are not strategically active. Our paper differs from AG and BB in two main aspects. First, in terms of application, while AG and BB apply to the situation in which a general outlet competes with a number of specialized (or local) outlets, our paper focuses on the competition between two general media with potentially different targeting abilities, such as

\footnotetext{
${ }^{1}$ In their model, an increase in targeting ability means that consumers with the same interest in products become more concentrated in fewer specialized markets.
} 
Google and Facebook, or Facebook and The New York Times. Second, in both AG and BB the media do not compete for consumers; the consumers are passive and do not incur any nuisance cost when viewing irrelevant ads. In our model, however, media competition for consumers is an important element. As we will show later, these differences lead to different predictions regarding the impact of targeting.

Based on Anderson and Coate (2005), we develop a model with two competing platforms acting as bridges between consumers and advertising firms. Consumers' tastes about the two platforms' contents are horizontally differentiated a la Hotelling. Advertising firms (simply firms sometimes) are heterogeneous in terms of the profitability of each product sold. While firms can be multi-homing, that is, they can participate on both platforms, consumers are single-homing, which means each consumer only participates on one platform. Consumers have heterogeneous tastes about firms' products, and the role of advertising is to turn potential matches (between consumers and firms) into actual purchases. For each consumer-firm pair on a platform, the platform is able to imperfectly identify whether the consumer is interested in (or relevant for) the firm's product. The accuracy of identifying relevant consumers for firms' products indicates the targeting ability of a platform, and the two platforms have different targeting abilities. Overall, consumers incur nuisance costs in viewing ads. In terms of timing, first the two platforms simultaneously set ad prices per impression. Then firms decide whether to participate on each platform, and at the same time consumers decide which platform to join.

We first show that there is a unique equilibrium. Our first set of main results illustrate how asymmetry in targeting ability translates into asymmetric behavior of the two platforms. First, compared to the disadvantaged platform (with the lower targeting ability), in equilibrium the advantaged platform (with the higher targeting ability) always has more advertising firms, attracts more consumers, and is more profitable. However, the advantaged platform could charge a higher or a lower ad price, and has a higher or lower total number of ads, than the other platform. Note that these predictions are potentially testable (we can interpret the platform with a higher targeting ability as an online platform, and the other as an offline platform). Second, consumers on the advantaged platform always incur a lower nuisance cost than those on the other platform; and any firm that advertises on both platforms earns a higher profit on the advantaged platform.

The driving force behind the above results are as follows. Given the negative externality imposed by advertising firms on consumers, each platform faces a tradeoff between the number of participating firms and the number of participating consumers. From a platform's point of view they are complements. Thus naturally the advantaged platform "spends" its advantage in accommodating more participating firms as well as attracting more consumers. The ambiguous conclusions on the price and total volume of ads result from two opposing effects: a mix effect 
(the advantaged platform having a lower proportion of irrelevant ads) and a volume effect (the advantaged platform having more advertising firms). Specifically, due to the mix effect, the advantaged platform tends to have a higher price and a smaller volume of ads than the disadvantaged one. But in the meantime, the volume effect works in the opposite direction: it leads to a lower ad price and a bigger ad volume on the advantaged platform. The overall effect can go either way.

Our second set of main results illustrate how an increase in the targeting ability of the advantaged platform affects the equilibrium outcome. First, the advantaged platform will have more advertising firms, attract more consumers, and become more profitable. The disadvantaged platform will have fewer advertising firms, attract fewer consumers, have fewer ads in total, increase its ad price, and become less profitable. However, the ad price of the advantaged platform could either increase or decrease, and the same pattern holds for its total volume of ads. $^{2}$ Again, these predictions are potentially testable. In particular, the predictions regarding the disadvantaged platform are largely consistent with the anecdotal evidence mentioned earlier: offline media have been suffering from the competition with new Internet media. Second, all consumers, on either platform, are unambiguously better off. Moreover, some less profitable firms, who were excluded previously but start to advertise on the advantaged platform after the change, are better off; all firms will be better off if their profitability follows a uniform or an exponential distribution. ${ }^{3}$

Some of the above predictions are different from those in AG and BB. In particular, when the targeting ability of the general outlet increases, AG show that the profit of the general outlet decreases but that of the local outlets increases. The prediction of our model is the opposite: when one platform's targeting ability increases, it always benefits that platform and hurts the other. When the targeting ability of the specialized markets increases, BB predict that the number of participating firms in each specialized market decreases, the advertising revenue of each market could increase or decrease, and the equilibrium ad prices are either hump-shaped or always decreasing in targeting ability. As mentioned earlier, these predictions differ from the results of this paper due to the differences in modeling. ${ }^{4}$

As to consumer welfare, Johnson (2013) shows that consumers might be worse off as the targeting ability of ads increases. In contrast, in our model consumers are always better off from an increase in targeting ability. ${ }^{5}$ The ambiguity result in Johnson (2013) is due to the

\footnotetext{
${ }^{2}$ The underlying reason for the ambiguity of the ad price and the total number of ads is that the mix effect and the volume effect work in opposite directions.

${ }^{3}$ With these distributions, the combined total number of relevant ads on the two platforms increases as well.

${ }^{4} \mathrm{In} \mathrm{BB}$, targeting abilities are market-specific and they are correlated across markets. In our model, targeting abilities are platform-specific. Another difference is that in BB the total volume of ads per consumer is always fixed (defined as the exogenous supply of ads), while in our model it is endogenous.

${ }^{5}$ We also show that, when the targeting ability of the disadvantaged platform increases, all consumers are still
} 
fact that the mix effect and the volume effect work in opposite directions, and either of them can dominate. The underlying difference is that in our model the platforms actively set the ads volume and are competing with each other. When one platform's targeting ability increases, this platform will control its ads volume so that it gains additional consumers, which implies that consumers will benefit or the mix effect dominates the volume effect. ${ }^{6}$

Finally, we compare social incentives and private incentives to invest in targeting abilities. In particular, two platforms are symmetric and they both make investment decisions in the first stage. It turns out that in equilibrium platforms could underinvest as well as overinvest in targeting ability. However, quantitatively underinvestment in targeting ability is much more likely to occur, while overinvestment occurs only under very special conditions.

This paper is related to the literature on informative advertising (Butters, 1977; Grossman and Shapiro, 1984). As mentioned before, an earlier literature on targeted advertising (Esteban et al., 2001; Iyer et al., 2005; Galeotti and Moraga-Gonzlez, 2008; and Gal-Or et al., 2010) focuses on how targeted advertising affects the competition and equilibrium prices in product markets, instead of analyzing the volume and price of advertising. Taylor (2011) not only studies the effect of targeting accuracy on competition in product markets, but also considers its impact on media's choices regarding content differentiation. ${ }^{7}$ In Zhang and Katona (2012), advertising firms again compete with each other in the product market, and they introduce a monopoly platform who controls the targeting accuracy of ads. In this context, they study how the degree of competition in the product market affects the platform's choice of targeting accuracy.

Kox et al. (2017) study targeted advertising with consumers having privacy concerns (dislike being tracked). ${ }^{8}$ They also adopt a framework of two-sided markets with competing platforms. They show that more targeting hurts platforms yet in equilibrium platforms choose maximum amount of targeting, which also hurts consumers due to lower privacy. However, different from our model, in their model consumers do not incur nuisance costs from viewing ads. Note that the direct impact of targeting is the opposite between two models: in their model more targeting directly hurts consumers while in our model it directly benefits consumers.

Our paper is also related to the rapidly expanding literature on competition in two-sided

better off. When the two platforms are symmetric (have the same targeting ability), consumers on either platform are again unambiguously better off when the targeting ability increases.

${ }^{6}$ More precisely, the other platform responds by reducing the number of advertising firms. Thus the consumers on the other platform benefit from fewer ads. This means that the consumers on the original platform must benefit even more since otherwise it would have lost some consumers.

${ }^{7}$ In a consumer search model, de Corniere (2016) studies how a search engine's targeting ability of keywords search affects the fee of advertising. Yang (2013) develops a model of targeted search, analyzing how the quality of search affects the variety of goods offered, prices, and consumer welfare.

${ }^{8}$ de Corniere and de Nijs (2016) also study online advertising with privacy. In their model, there is a single platform and privacy is modeled as revealing consumers' personal information to relevant advertising firms. However, consumers do not have intrinsic preferences over privacy. 
markets (see Rochet and Tirole, 2003, 2006; and Armstrong, 2006). The most relevant model to our paper is the model of "competitive bottlenecks" (Armstrong, 2006), in which one side of the market is single-homing, while the other is multi-homing. The major insight of Armstrong (2006) is that, due to the competitive pressure on the single-homing side, platforms might be forced to transfer some of the surplus towards this side, which leads to too few multi-homing agents in equilibrium.

Finally, our paper is related to the literature on media competition. Dukes (2004) studies a model of media competition in which advertising firms directly compete in the product market. He investigates how the degree of product differentiation among firms and the degree of media differentiation affect the equilibrium level of advertising. Our paper is different form his in several aspects. First, in our model firms do not compete with each in the product market. Second, in his model ads do not impose nuisance costs on consumers. And finally, in his model ads are not targeted.

In terms of modeling, our paper is closely related to Masson et al. (1990) and Anderson and Coate (2005), who study advertising on competing (media) platforms and ads impose negative externalities on consumers. The difference is that in their models two platforms are symmetric and advertising cannot be targeted. In a media competition model, Peitz and Valletti (2008) introduce the possibility that consumers may pay for their access to content, and the results show that advertising intensity is higher in free-to-air television than in pay-tv stations. Ambrus and Reisinger (2006) extend Anderson and Coate (2005), by allowing consumers/viewers to be multihoming, and then compare this to the original setting with single-homing consumers. Athey et al. (2013) extend Anderson and Coate (2005) to a setting in which consumers switch media outlets stochastically (multi-homing, but not fully), and study how the tracking technologies of platforms affect the equilibrium outcomes in the advertising market. None of these papers studies targeted advertising.

The rest of the paper is organized as follows. Section 2 sets up the model. In Section 3 we characterize the equilibrium outcome. Section 4 studies the impacts of targeting. In Section 5 we endogenize the targeting abilities of platforms. Section 6 offers conclusions and discussions. All the proofs can be found in the Appendix.

\section{Model}

There are two platforms, $A$ and $B$, who bring together consumers and advertising firms (simply firms sometimes). Consumers consume the content of a platform, and firms participate on platforms in order to send ads to consumers. Both consumers and firms are of unit mass. ${ }^{9}$ The

\footnotetext{
${ }^{9}$ It is not essential that consumers and firms are of the same measure.
} 
two platforms' contents are horizontally differentiated. Using Hotelling's location model, we assume that platforms $A$ and $B$ are located at 0 and 1, respectively. Consumers' tastes about the platforms' contents are also differentiated. Specifically, consumers are uniformly distributed on $[0,1]$. Let $d$ be the location of a consumer. Consumers are single homing, meaning that a consumer will join only one platform. Firms are potentially multi-homing: each firm can join neither, one, or both platforms.

For any given consumer-firm pair, with probability $q \in(0,1 / 2)$ there is a match. That is, the consumer is interested in the firm's product, and we call such a consumer a relevant consumer, and an ad between such a pair a relevant ad. The probability that a match exists is i.i.d. across all consumer-firm pairs. A consumer will buy one unit of product from a firm if and only if the consumer is relevant to the firm and he receives an ad from that firm. So in our model advertising is purely informative. Denote $S \in\{0,1\}$ as the state indicating whether a consumer is relevant to a firm, with 1 (0) denoting that the consumer is relevant (irrelevant). For any consumer-firm pair on platform $i$, the platform generates a signal $s \in\{0,1\}$ regarding the possible match. The signals are also conditionally independent across consumer-firm pairs. The information structure is as follows:

$$
\operatorname{Pr}\{s=1 \mid S=1\}=\alpha_{i} ; \operatorname{Pr}\{s=0 \mid S=0\}=\alpha_{i} .
$$

The parameter $\alpha_{i} \in(1 / 2,1]$, which measures the accuracy of the signals, captures the targeting ability of platform $i$. We assume that $\alpha_{A}$ and $\alpha_{B}$ are common knowledge. The posteriors that a consumer is relevant to a firm can be calculated as follows:

$$
\begin{aligned}
& \operatorname{Pr}\{S=1 \mid s=1\}=\frac{\alpha_{i} q}{\alpha_{i} q+\left(1-\alpha_{i}\right)(1-q)} \\
& \operatorname{Pr}\{S=1 \mid s=0\}=\frac{\left(1-\alpha_{i}\right) q}{\left(1-\alpha_{i}\right) q+\alpha_{i}(1-q)} .
\end{aligned}
$$

We assume $q<1 / 2$ is small enough such that $\operatorname{Pr}\{S=1 \mid s=0\}$ is also small enough, so that it never pays for any firm to send ads to consumers when the signal is 0 . For any firm, the set of consumers with signal 1 can be considered as that firm's targeted set of consumers, to which the firm might send ads. Note that the size of the targeted set of consumers is $\alpha_{i} q+\left(1-\alpha_{i}\right)(1-q)$, which is increasing in $q$, and is decreasing in $\alpha_{i}$ as $q<1 / 2$. Also note that, although the targeted sets of consumers are different for different firms, for each firm on the same platform the targeted set of consumers is of the same size, since the probability of being relevant is i.i.d. across consumer-firm pairs and the signals are conditionally independent. The two platforms are different in targeting abilities. Specifically, $\alpha_{B}>\alpha_{A}$. One can consider platform $A$ as a traditional offline media and platform $B$ as an online media. In case that both platforms are online media, the difference in targeting abilities could result from different technologies in tracing consumers. 
Each consumer incurs a nuisance cost $\gamma>0$ by viewing an ad. And each consumer gets a gross payoff $\lambda>0$ if she buys a relevant product. ${ }^{10}$ With this specification, a consumer incurs a net nuisance cost of $\gamma$ by viewing an irrelevant ad, and a net nuisance cost of $\gamma-\lambda$ by viewing a relevant ad (could be net benefit if $\lambda>\gamma$ ). A location- $d$ consumer gets a utility of $\bar{\beta}-t d$ minus the net nuisance cost of ads if participating on platform $A$, and she gets a utility of $\bar{\beta}-t(1-d)$ minus the net nuisance cost of ads if participating on platform $B$. The parameter $\bar{\beta}$ captures the gross utility of a consumer joining either platform by consuming the content provided by that platform. We assume $\bar{\beta}$ is high enough so that all consumers participate. The parameter $t$ is the transportation cost in standard Hotelling models, which indicates the degree of horizontal differentiation between two platforms' contents.

Firms are heterogeneous in terms of profitability. Denote $v$ as a firm's profit per sale. Firms' types $v$ are distributed on $[0, \bar{v}]$ with cumulative distribution function $F(v)$ and density function $f(v)$, with $f(\cdot)>0$ everywhere in the support, differentiable, and strictly logconcave.

The timing is as follows. In the first stage, the two platforms set advertising prices (per impression), $p_{A}$ and $p_{B}$, simultaneously. In the second stage, observing $p_{A}$ and $p_{B}$, consumers simultaneously decide which platform to join, and firms, at the same time, decide whether to participate on each platform simultaneously. All agents have rational expectations.

Our model is based on Anderson and Coate (2005). The difference is that we add the aspect of advertisement targeting, and two platforms are asymmetric in that they have different targeting abilities. The model also resembles Armstrong's (2006) model of "competitive bottlenecks," in which platforms are actively competing for single-homing consumers, while there is no competition for multi-homing firms. The two-sided market only has an one-way externality: firms exert externalities on consumers by posting ads.

Several real-world examples largely fit the main assumptions of our model. In the market of social media, Facebook and Myspace compete for users and they begin to rely more heavily on advertising revenue. Moreover, users are typically single-homing. In the magazine market, Time and The Economist are competing for readers. In the local news markets, local newspapers and local TV stations are competing for viewers. In the latter two examples, some consumers might be multi-homing. But it does not qualitatively affect the results of our model as long as some consumers are single-homing (as if platforms only compete for the single-homing consumers). Even if all consumers are multi-homing, it is reasonable to think that each consumer's attention allocation between two media is sensitive to the amount of irrelevant ads on them: other things equal, a consumer tends to spend more time on a medium with less irrelevant ads. This model of multi-homing consumers with attention allocation is quite similar to our model with single-homing consumers, as in both cases platforms are competing for consumer attention by

\footnotetext{
${ }^{10}$ Johnson (2013) makes a similar assumption. $\lambda$ can be considered as the surplus enjoyed by a consumer engaging in trade.
} 
restricting the amount of ads.

\section{Equilibrium with Competing Platforms}

To characterize equilibrium, we first analyze firms' behavior. Given an ad price $p_{i}, i=A, B$, a firm of type $v$ will get the following profit per ad if advertising on platform $i: \frac{\alpha_{i} q v}{\alpha_{i} q+\left(1-\alpha_{i}\right)(1-q)}-p_{i}$. Thus a firm of type $v$ will advertise on platform $i$ if and only if

$$
v \geq \frac{\alpha_{i} q+\left(1-\alpha_{i}\right)(1-q)}{\alpha_{i} q} p_{i} \equiv \widehat{v}_{i} .
$$

The term $\widehat{v}_{i}$ is the cutoff or marginal type of firms for platform $i$. Given that firms are multihoming, a firm's decision to join platform $i$ is independent of its decision to join platform $j$. Moreover, since firms pay prices per impression, a firm's decision as to whether to join either platform does not depend on consumers' platform choices. Let $\mu_{i}=1-F\left(\widehat{v}_{i}\right)$ be the fraction of firms that advertise on platform $i$. Note that setting the ad price $p_{i}$ is equivalent to choosing the fraction of participating firms $\mu_{i}$. Since working with $\mu_{i}$ is more convenient, in later analysis we will use $\mu_{i}$ instead of $p_{i}$ as platform $i$ 's strategy. In particular, the relationship between $p_{i}$ and $\mu_{i}$ can be expressed as follows:

$$
p_{i}\left(\mu_{i}\right)=\frac{\alpha_{i} q F^{-1}\left(1-\mu_{i}\right)}{\alpha_{i} q+\left(1-\alpha_{i}\right)(1-q)} .
$$

Clearly, a higher price $p_{i}$ leads to a smaller fraction of participating firms $\mu_{i}$.

Now we analyze consumers' behavior. Let $m_{i}$ be the fraction of consumers joining platform $i$. Sometimes we call $m_{i}$ the consumer base of platform $i$. Recall that consumers are single-homing. Because potential matches are i.i.d. and signals are conditionally i.i.d. across all consumer-firm pairs, all consumers joining platform $i$ will receive the same number of ads. A consumer on platform $i$ incurs the following expected net nuisance cost, $y_{i}$, by viewing each ad: ${ }^{11}$

$$
y_{i}=\gamma(1-q)\left(1-\alpha_{i}\right)-(\lambda-\gamma) \alpha_{i} q .
$$

And the total net nuisance cost a consumer on platform $i$ incurs is $y_{i} \mu_{i}$. Each consumer chooses the platform that minimizes the sum of his transportation cost and the total net nuisance cost. This leads to the following expressions for the consumer bases, ${ }^{12}$

$$
\begin{aligned}
& m_{A}\left(\mu_{A}, \mu_{B}\right)=\frac{1}{2}+\frac{\gamma(1-q)}{2 t}\left[\left(1-\alpha_{B}\right) \mu_{B}-\left(1-\alpha_{A}\right) \mu_{A}\right]-\frac{(\lambda-\gamma) q}{2 t}\left[\alpha_{B} \mu_{B}-\alpha_{A} \mu_{A}\right], \\
& m_{B}\left(\mu_{A}, \mu_{B}\right)=\frac{1}{2}+\frac{\gamma(1-q)}{2 t}\left[\left(1-\alpha_{A}\right) \mu_{A}-\left(1-\alpha_{B}\right) \mu_{B}\right]-\frac{(\lambda-\gamma) q}{2 t}\left[\alpha_{A} \mu_{A}-\alpha_{B} \mu_{B}\right] .
\end{aligned}
$$

\footnotetext{
${ }^{11}$ For each firm on platform $i$, with probability $(1-q)\left(1-\alpha_{i}\right)\left(\alpha_{i} q\right)$ the consumer will be in that firm's targeted set (signal is 1 for the consumer-firm pair) and the consumer is actually irrelevant (relevant) for the firm's product.

${ }^{12}$ Recall that firms and consumers move simultaneously. But this does not matter as consumers have rational expectations: given the ad prices, consumers can predict how many firms will advertise on each platform and how many ads will be sent on each platform.
} 
Let $x_{i} \equiv y_{i} / 2 t$. Then the consumer bases can be more compactly written as

$$
m_{i}\left(\mu_{i}, \mu_{j}\right)=\frac{1}{2}+x_{j} \mu_{j}-x_{i} \mu_{i} .
$$

The term $x_{i}$ captures how sensitive the consumer base is to changes in $\mu_{i}$ : bigger $x_{i}$ and $x_{j}$ (a bigger net nuisance cost and a smaller transportation cost $t$ ) imply stronger competition for consumers. By $q<1 / 2$ and $\alpha_{A}<\alpha_{B}$, it can be easily verified that $y_{A}>y_{B}$ and $x_{A}>$ $x_{B}$. Intuitively, since platform $B$ has a higher targeting ability, a consumer on platform $B$ in expectation incurs a lower net nuisance cost from viewing an ad than a consumer on platform $A$.

Condition $1 y_{B}=-(\lambda-\gamma) q \alpha_{B}+\gamma(1-q)\left(1-\alpha_{B}\right)>0$.

For the rest of the paper, we assume that Condition 1 holds. Condition 1 says that the net expected nuisance cost of viewing an ad for a consumer on platform $B$ is positive. Since $\alpha_{B}>\alpha_{A}$, it implies that, $y_{A}$, the net expected nuisance cost of viewing an ad for a consumer on platform $A$, is positive as well. It further implies that $x_{A}$ and $x_{B}$ are both positive. Intuitively, Condition 1 means that ads overall impose negative externalities on consumers. ${ }^{13}$ We believe Condition 1 is a reasonable assumption, as in many real-world applications ads by large impose negative externalities on consumers. This is also consistent with what is commonly assumed in the literature (Anderson and Coate, 2005, for instance).

Now we analyze platforms' behavior. Platform $i$ 's profit, $\Pi_{i}\left(\mu_{i}, \mu_{j}\right)$, can be computed as

$$
\Pi_{i}\left(\mu_{i}, \mu_{j}\right)=\left[\alpha_{i} q+\left(1-\alpha_{i}\right)(1-q)\right] p_{i}\left(\mu_{i}\right) m_{i}\left(\mu_{i}, \mu_{j}\right) \mu_{i} .
$$

The term $m_{i} \mu_{i}$ is the total number of consumer-firm pairs on platform $i$. Among those, a $\alpha_{i} q+\left(1-\alpha_{i}\right)(1-q)$ fraction of consumer-firm pairs have signal 1 and ads will be sent. Therefore, the total number of impressions is $\alpha_{i} q+\left(1-\alpha_{i}\right)(1-q)$ times $m_{i} \mu_{i}$. Multiplying by the ad price per impression $p_{i}$, we get the above expression of $\Pi_{i}$. An important observation from the above expression is that, for each platform, the fraction of advertising firms $\mu_{i}$ and its consumer base $m_{i}$ are complements, as $\Pi_{i}$ is proportional to $m_{i} \mu_{i}$. Using (1) to get rid of $p_{i}$, the profit function can be written as

$$
\Pi_{i}\left(\mu_{i}, \mu_{j}\right)=\alpha_{i} q F^{-1}\left(1-\mu_{i}\right) m_{i}\left(\mu_{i}, \mu_{j}\right) \mu_{i}=\alpha_{i} q \mu_{i} \widehat{v}_{i} m_{i}\left(\mu_{i}, \mu_{j}\right) .
$$

In (4), the term $\alpha_{i} q \mu_{i} \widehat{v}_{i} \equiv \alpha_{i} q R\left(\mu_{i}\right)$ is platform $i$ 's revenue per consumer. ${ }^{14}$

\footnotetext{
${ }^{13}$ Note that Condition 1 is always satisfied if $\lambda<\gamma$. If $\lambda>\gamma$, then it requires either $q$ be small enough or $\alpha_{B}$ be sufficiently below 1 .

${ }^{14}$ The assumption that $f$ is logconave is standard in the literature. This is to ensure that the demand function for ads is well behaved so that platforms' revenue function $R(\mu)$ is concave in $\mu$. Many common distributions, such as uniform distribution, exponential distribution, and normal (or truncated normal) distribution, have logconcave density functions (see Bagnoli and Bergstrom, 2005, for details).
} 
Denote an equilibrium as $\left(\mu_{A}^{*}, \mu_{B}^{*}\right)$. Differentiating $\Pi_{i}\left(\mu_{i}, \mu_{j}\right)$ (equation (4)) with respect to $\mu_{i}$, we get

$$
\frac{\partial \Pi_{i}}{\partial \mu_{i}}=\alpha_{i} q\left\{\left[\widehat{v}_{i}-\frac{1-F\left(\widehat{v}_{i}\right)}{f\left(\widehat{v}_{i}\right)}\right] m_{i}\left(\mu_{i}, \mu_{j}\right)-x_{i} \widehat{v}_{i}\left[1-F\left(\widehat{v}_{i}\right)\right]\right\} .
$$

According to (5), an increase in $\mu_{i}$ affects $\Pi_{i}$ through two channels. First, it affects the marginal revenue per consumer, which is captured by the term $R^{\prime}\left(\mu_{i}\right)=\widehat{v}_{i}-\frac{1-F\left(\widehat{v}_{i}\right)}{f\left(\widehat{v}_{i}\right)}$. Second, it will change platform $i$ 's consumer base (the business stealing effect), which is captured by the second term in (5). By the logconcavity of $f$, the marginal revenue function $R^{\prime}(\mu)$ is strictly decreasing in $\mu .{ }^{15}$ Moreover, $R^{\prime}(\bar{v})=\bar{v}-\frac{1-F(\bar{v})}{f(\bar{v})}>0$ and $R^{\prime}(0)=0-\frac{1-F(0)}{f(0)}<0$. Thus there is a unique $\widetilde{\mu} \in(0,1)$ such that $R(\widetilde{\mu})=0$. Note that $\widetilde{\mu}$ uniquely maximizes revenue per consumer $R(\mu)$, and a monopoly platform will choose $\widetilde{\mu}$.

Lemma 1 (i) $\frac{\partial \Pi_{i}}{\partial \mu_{i}}<0$ if $\mu \in(\widetilde{\mu}, 1]$. (ii) $\mu_{i}^{*} \in[0, \widetilde{\mu}]$. (iii) For $\mu_{i} \in[0, \widetilde{\mu}), \frac{\partial^{2} \Pi_{i}}{\partial \mu_{i}^{2}}<0$ and $\frac{\partial^{2} \Pi_{i}}{\partial \mu_{i} \partial \mu_{j}}>0$.

By part (ii) of Lemma 1, in search for equilibrium we can restrict our attention to the domain $[0, \widetilde{\mu}]$. The underlying reason is that the business stealing effect is always negative: an increase in $\mu_{i}$ reduces the consumer base of platform $i$. This means that to satisfy the first-order condition, the marginal revenue per consumer has to be positive. Part (iii) shows that, in the relevant domain the second-order condition is satisfied, which implies that the first-order condition is sufficient for the best response function $\mu_{i}^{*}\left(\mu_{j}\right)$. The fact that $\frac{\partial^{2} \Pi_{i}}{\partial \mu_{i} \partial \mu_{j}}>0$ implies that $\mu_{A}$ and $\mu_{B}$ are strategic complements.

Based on previous analysis, an equilibrium $\left(\mu_{A}^{*}, \mu_{B}^{*}\right)$ is characterized by the following firstorder conditions:

$$
\begin{aligned}
& x_{B} \mu_{B}^{*}=x_{A} \mu_{A}^{*}+\frac{x_{A} \mu_{A}^{*} \widehat{v}_{A}^{*}}{\widehat{v}_{A}^{*}-\frac{1-F\left(\widehat{v}_{A}^{*}\right)}{f\left(\widehat{v}_{A}^{*}\right)}}-\frac{1}{2} ; \\
& x_{A} \mu_{A}^{*}=x_{B} \mu_{B}^{*}+\frac{x_{B} \mu_{B}^{*} \widehat{v}_{B}^{*}}{\widehat{v}_{B}^{*}-\frac{1-F\left(\widehat{v}_{B}^{*}\right)}{f\left(\widehat{v}_{B}^{*}\right)}}-\frac{1}{2} .
\end{aligned}
$$

Lemma 2 There is a unique equilibrium.

\section{The Impacts of Targeting}

\subsection{Comparison between two platforms}

After establishing the existence and uniqueness of equilibrium, we compare the two platforms' equilibrium behavior in the following proposition (how the asymmetry in targeting abilities

\footnotetext{
${ }^{15}$ Since $f$ is logconcave, $\frac{1-F(v)}{f(v)}$ is strictly decreasing in $v$ (see Bagnoli and Bergstrom, 2005, for details). Thus $v-\frac{1-F(v)}{f(v)}$ is strictly increasing in $v$, which implies that $R^{\prime}(\mu)$ is strictly decreasing in $\mu$.
} 
translates into asymmetric outcomes).

Proposition 1 In the unique equilibrium the following properties hold. (i) Compared to platform $A$, platform $B$ has more participating firms, a bigger consumer base, and a higher equilibrium profit: $\mu_{B}^{*}>\mu_{A}^{*}, m_{A}\left(\mu_{A}^{*}, \mu_{B}^{*}\right)<1 / 2<m_{B}\left(\mu_{A}^{*}, \mu_{B}^{*}\right)$, and $\Pi_{A}^{*}<\Pi_{B}^{*}$. (ii) Platform $B$ charges a higher ad price $\left(p_{A}^{*}<p_{B}^{*}\right)$ if $\frac{1+\frac{1-\alpha_{A}}{\alpha_{A}} \frac{1-q}{q}}{1+\frac{1-\alpha_{B}}{\alpha B} \frac{1-q}{q}} \geq \frac{y_{A}}{y_{B}}$. (iii) Platform $B$ has less ads in total if both $x_{A}$ and $x_{B}$ are close enough to 0.

The results in part (i) of Proposition 1 are intuitive. Since platform $B$ has a higher targeting ability, each ad on platform $B$ imposes less negative externality on consumers $\left(y_{B}<y_{A}\right.$ and hence $x_{B}<x_{A}$ ). This means the consumer base is less sensitive to $\mu_{B}$ than to $\mu_{A}$. As a result, in equilibrium platform $B$ should have a lower marginal revenue per ad, which implies more participating firms. However, in equilibrium, platform $B$, who has a natural advantage in attracting more consumers, will not accommodate too many firms such that its consumer base falls below $1 / 2$. This is because, from each platform's point of view, the number of participating firms and the number of participating consumers are complements. Thus, in equilibrium platform $B$ has a bigger consumer base. The fact that platform $B$ has a higher profit is easy to understand: by mimicking platform $A$ 's strategy, platform $B$ can always guarantee a higher profit than platform $A$ 's since it will have a bigger consumer base.

However, whether platform $B$ will charge a higher ad price or have more total ads, relative to platform $A$, is ambiguous. This is because there are two effects working in opposite ways. We call the first effect the mix effect and the second one the volume effect. Specifically, the mix effect is due to the fact that on platform $B$ ads are more "accurate" or more relevant for consumers, while the volume effect refers to the fact that on platform $B$ there are more advertising firms $\left(\widehat{v}_{A}^{*}>\widehat{v}_{B}^{*}\right)$. Regarding the ad price, the mix effect implies that platform $B$ can charge a higher ad price to attract the same cutoff type of firms, as fewer ads are sent to irrelevant consumers and thus are wasted (generate no sale for firms). On the other hand, the volume effect tends to make the price charged by platform $B$ relatively lower, as the marginal type of firms is lower on platform $B\left(\widehat{v}_{A}^{*}>\widehat{v}_{B}^{*}\right){ }^{16}$ The overall effect can go either way. Regarding the total number of ads, the volume effect (more participating firms on platform $B$ ) tends to increase the total number of ads on platform $B$ relative to platform $A$. However, the mix effect means that the proportion of irrelevant ads on platform $B$ is smaller, which tends to decrease the total number of ads on platform $B$ relative to platform $A$. Again, the overall effect can go either way. ${ }^{17}$

\footnotetext{
${ }^{16}$ In general, the volume effect is hard to quantify, as it depends on how sensitive $\widehat{v}_{A}^{*}$ and $\widehat{v}_{B}^{*}$ are to $\alpha_{A}$ and $\alpha_{B}$, which in turn depends on the distribution of firm types.

${ }^{17}$ However, we can show unambiguously that platform $B$ has more relevant ads in total than platform $A$. This is because platform $B$ has more participating firms, a bigger consumer base, and a higher targeting ability, which means more relevant consumers are identified.
} 
Regarding the ad price, part (ii) of Proposition 1 identifies a sufficient condition for the mix effect to dominate the volume effect. The LHS of the condition is the ratio of the signal to noise ratio on platform $B$ to that on platform $A$, which roughly measures the strength of the mix effect. The RHS of the condition is the ratio of the negative externality imposed on consumers by each ad on platform $A$ to that on platform $B$. This roughly measures the strength of the volume effect, as it cannot be surpassed by the ratio of the number of participating firms on platform $B$ to that on platform $A$. Thus, the condition specifies a scenario in which the mix effect dominates. Here we provide an example in which $p_{A}^{*}>p_{B}^{*}$ and the volume effect dominates. Suppose $v$ is uniformly distributed on $[0,1], q=0.3, t=0.1, \gamma=3, \lambda=2.9, \alpha_{A}=0.88$, and $\alpha_{B}=0.98$. Then $0.6062=p_{A}^{*}>p_{B}^{*}=0.5440$. Part (iii) of Proposition 1 identifies a condition under which platform $A$ has more ads in total. ${ }^{18}$

The predictions of Proposition 1 are potentially empirically testable. Using the interpretation of an online platform $(B)$ competing with an offline one $(A)$, Proposition 1 implies that the online platform will have more advertising firms, attract more consumers, and is more profitable, relative to the offline platform. Moreover, relatively low-profit firms will only advertise on the online platform. However, the online platform could charge a higher or a lower ad price than the offline platform, and the total number of ads could be higher or lower on the online platform relative to the offline one.

The next proposition compares consumer welfare and firms' profits across two platforms.

Proposition 2 In the unique equilibrium the following properties hold. (i) Compared to consumers on platform $A$, each consumer on platform B incurs a lower total net nuisance cost. (ii) Any firm that advertises on both platforms earns a higher profit on platform $B$.

Part (i) of Proposition 2 is surprising. Note that the mix effect favors consumers on platform $B$, as its proportion of irrelevant ads is smaller. However, the volume effect favors consumers on platform $A$, since it has fewer participating firms. Nevertheless, Consumers on platform $B$ always incur a lower total net nuisance cost than those on platform $A$, which means that the mix effect dominates. Actually this result is directly implied by the fact that platform $B$ has a bigger equilibrium consumer base. If consumers on platform $B$ were incurring a higher total net nuisance cost than those on platform $A$, then platform $B$ would have a smaller consumer base than platform $A$. More fundamentally, this result is due to the fact, for each platform, the number of participating firms and the number of participating consumers are complements. Thus, in equilibrium platform $B$ controls the size of the volume effect such that it still has a bigger consumer base than platform $A$.

\footnotetext{
${ }^{18}$ In the limit, when both $x_{A}$ and $x_{B}$ go to 0 (this is the case when both $\gamma$ and $\lambda$ converge to 0 ), both platforms act like local monopolists and both accommodate the same number of firms, $\widetilde{\mu}$. In this limiting case, the volume effect disappears and the mixed effect implies that platform $A$ has more ads in total.
} 
Part (ii) of Proposition 2 is easy to understand. Since platform $B$ has more participating firms and a lower marginal firm, each firm on platform $B$ must earn a higher profit per consumer than it can earn on platform $A$. Each participating firm's total profit should also be higher on platform $B$ as it has a bigger consumer base. To summarize, all parties on platform $B$ (consumers, firms, and the platform itself) benefit from its higher targeting ability relative to the corresponding parties on platform $A$.

Since firms' ads impose negative externalities on consumers, an increase in the nuisance cost $\gamma$, just like a decrease in the transportation cost $t$, means the competition for consumers becomes fiercer. As a result, both platforms reduce ad volume per consumer by accommodating fewer firms. The profit of platform $A$ decreases as $\gamma$ increases. However, as $\gamma$ increases the profit of platform $B$ could either decrease or increase. This is because, although platform $B$ suffers from intensified competition, the increase in $\gamma$, in the mean time, amplifies its advantage in attracting consumers. ${ }^{19}$

\subsection{Increases in targeting abilities}

Now we investigate how changes in platforms' targeting abilities affect the equilibrium outcome. We begin with an increase in platform $B$ 's targeting ability.

Proposition 3 Suppose $\alpha_{B}^{\prime}>\alpha_{B}$ and $\alpha_{A}$ remains the same. Let the superscript' denote the endogenous variables in the equilibrium under $\left(\alpha_{A}, \alpha_{B}^{\prime}\right)$. Then, (i) the number of advertising firms on platform $A$ decreases, and that on platform $B$ increases: $\mu_{A}^{* \prime}<\mu_{A}^{*}$ and $\mu_{B}^{* \prime}>\mu_{B}^{*}$; (ii) platform $A$ 's consumer base shrinks while Platform $B$ 's consumer base expands: $m_{A}^{* \prime}<m_{A}^{*}$ and $m_{B}^{* \prime}>m_{B}^{*}$; (iii) platform $A$ 's profit decreases while platform $B$ 's profit increases: $\Pi_{A}^{* \prime}<\Pi_{A}^{*}$ and $\Pi_{B}^{* \prime}>\Pi_{B}^{*}$. (iv) platform $A$ 's ad price increases, $p_{A}^{* \prime}>p_{A}^{*}$, while platform $B$ 's ad price could either increase or decrease; in particular, $p_{B}^{* \prime}>p_{B}^{*}$ if $\frac{1+\frac{1-\alpha_{B}}{\alpha_{B}} \frac{1-q}{q}}{1+\frac{1-\alpha_{B}^{\prime}}{\alpha_{B}^{\prime}} \frac{1-q}{q}} \geq \frac{y_{B}}{y_{B}^{\prime}}$; (v) the total number of ads on platform $A$ decreases; the total number of ads on platform $B$ could increase or decrease; if the distribution of $v$ is either uniform or exponential, then the total combined relevant ads on two platforms increase.

The intuition for Proposition 3 is as follows. As the targeting ability of the advantaged platform $(B)$ increases, platform $B$ becomes more advantaged. Thus, naturally platform $B$ 's profit increases while that of platform $A$ decreases. Since ad revenue per consumer and consumer base are complements, platform $B$ will "spend" the additional advantage in increasing both the

\footnotetext{
${ }^{19}$ The following example shows that the profit of platform $B$ could increase in $\gamma$. Suppose $v$ is uniformly distributed on $[0,1], q=0.1, t=0.3, \lambda=0.5, \alpha_{A}=0.6$, and $\alpha_{B}=0.8$. As $\gamma$ increases from 0.2 to $0.6, m_{B}^{*}$ increases from 0.5329 to 0.5594 , and $\Pi_{B}^{*}$ increases from 0.0107 to 0.0111 . However, as $\gamma$ increases from 1.5 to 2 , $m_{B}^{*}$ decreases from 0.5557 to 0.5462 , and $\Pi_{B}^{*}$ decreases from 0.0104 to 0.0098 .
} 
fraction of participating firms and its consumer base (which means that the increase in the number of participating firms is low relative to the increase in targeting ability). To protect its own consumer base, platform $A$ responds by reducing the fraction of participating firms.

The result that platform $A$ increases its ad price when $\alpha_{B}$ increases seems somewhat counterintuitive, as one would expect platform $A$ to reduce its price when the other platform becomes more advantaged. However, in the current model platforms are not competing for firms, instead they are competing for consumers who suffer from negative externalities from firms' ads. As $\alpha_{B}$ increases, platform $A$ responds by reducing its fraction of participating firms. This means the marginal advertising firm of platform $A$ now has a higher profitability, which implies a higher ad price. The fact that the ad price of platform $B$ is not monotonic in $\alpha_{B}$ is again due to two opposite effects. In particular, according to the mix effect, a higher targeting ability enables platform $B$ to charge a higher ad price for the same marginal firm. On the other hand, an increase in $\alpha_{B}$ triggers a volume effect: platform $B$ accommodates more firms, which implies a downward shift in the type of the marginal firm and a lower ad price. The overall effect can go either way. In the proposition, we identified a sufficient condition for the mix effect to dominate. Roughly speaking, the LHS measures to what extent an increase in $\alpha_{B}$ improves the signal to noise ratio on platform $B$, the size of the mix effect. Similarly, the RHS measures to what extent an increase in $\alpha_{B}$ reduces the negative externalities imposed on consumers by each ad on platform $B$, which provides an upper bound for the size of the volume effect. ${ }^{20}$ As shown in the following numerical example, with a relatively big $\gamma, p_{B}^{*}$ could be increasing in $\alpha_{B}$ when $\alpha_{B}$ is relatively small and be decreasing in $\alpha_{B}$ when $\alpha_{B}$ is close to 1 . Suppose $v$ is uniformly distributed on $[0,1], q=0.3, t=0.3, \gamma=6, \lambda=5.9$, and $\alpha_{A}=0.85$. As $\alpha_{B}$ increases from 0.86 to $0.9, p_{B}^{*}$ increases from 0.5136 to 0.5213 . As $\alpha_{B}$ increases from 0.95 to $0.99, p_{B}^{*}$ decreases from 0.5170 to 0.5100 .

Part (v) of Proposition 3 shows that, while the total number of ads on platform $A$ decreases, the change in the total number of ads on platform $B$ is ambiguous. ${ }^{21}$ The non-monotonicity of the total number of ads on platform $B$ is again caused by the two countervailing effects. As $\alpha_{B}$ increases, the mix effect tends to reduce the number of irrelevant ads on platform $B$, while the volume effect tends to increase the number of ads. The following examples show that either effect could dominate: the total number of ads on platform $B$ is increasing in $\alpha_{B}$ when $\alpha_{B}$ is relatively small and is decreasing in $\alpha_{B}$ when $\alpha_{B}$ is close to 1 . Suppose $v$ is uniformly distributed on $[0,1], q=0.1, t=0.3, \gamma=4, \lambda=3.9$, and $\alpha_{A}=0.8$. As $\alpha_{B}$ increases from 0.82 to 0.9 , the total number of ads on platform $B$ increases from 0.0350 to 0.0380 . As $\alpha_{B}$ increases from 0.96

\footnotetext{
${ }^{20}$ In two empirical studies, Chandra (2009) on newspapers and Chandra and Kaiser (2010) on magazines, the ad prices are found to be higher in markets with more homogeneous subscribers (more segmented or a higher targeting ability).

${ }^{21}$ Note that on platform $A$ both the number of participating firms and the consumer base decrease.
} 
to 0.99 , the total number of ads on platform $B$ decreases from 0.0383 to 0.0365 .

As to the combined total number of relevant ads, there are two opposite effects as well. As $\alpha_{B}$ increases, the total number of relevant ads on platform $A$ decreases, while that on platform $B$ increases. $^{22}$ Moreover, platform $B$ gains market share, which tends to increase the combined total number of relevant ads. For general distributions of $v$, it is hard to compare the relative magnitudes of the first and the second effect, as how sensitive $\mu_{A}^{*}$ and $\mu_{B}^{*}$ are to $\alpha_{B}$ depends on the specific distribution. When the distribution is either uniform or exponential, we are able to show that the combined total number of relevant ads is increasing in $\alpha_{B}$.

Note that most of the predictions of Proposition 3 are potentially empirically testable. Again we use the interpretation of an online platform $(B)$ competing with an offline one $(A)$. Proposition 3 predicts that, as the targeting ability of the online platform increases, the online platform will have more advertising firms, attract more consumers, and become more profitable. The offline platform will have fewer advertising firms, attract fewer consumers, have fewer ads in total, increase its ad price, and become less profitable (these are largely consistent with the anecdotal evidence mentioned in the introduction). However, the ad price of the online platform could either increase or decrease, and the same pattern holds for the total volume of ads on the online platform.

Proposition 4 Suppose $\alpha_{B}^{\prime}>\alpha_{B}$ and $\alpha_{A}$ remains the same. Let the superscript' denote the endogenous variables in the equilibrium under $\left(\alpha_{A}, \alpha_{B}^{\prime}\right)$. Then, (i) each consumer incurs a lower total net nuisance cost; (ii) firms with $v \in\left(\widehat{v}_{B}^{* \prime}, \widehat{v}_{A}^{*}\right]$ are strictly better off; if the distribution of $v$ is either uniform or exponential, then firms participating on both platforms $\left(v \geq \widehat{v}_{A}^{* \prime}\right)$ are also better off.

Part (i) of Proposition 4 indicates that each consumer, on either platform, is unambiguously better off from an increase in $\alpha_{B}$. Intuitively, as $\alpha_{B}$ increases, platform $A$ reduces ad volume per consumer, and thus consumers on platform $A$ are better off. Since platform $B$ gains more consumers, consumers remaining with platform $B$ must gain more than those on platform $A$, because otherwise platform $B$ would have lost consumers to platform $A$.

This result is different from Johnson (2013), in which consumers might incur a higher total nuisance cost when the targeting ability of an implicit monopolist platform increases. In his model, an increase in targeting ability also has two effects: the mix effect benefiting consumers as the fraction of irrelevant ads decreases, and the volume effects hurting consumers as firms will send more ads. While in his model the overall effect is ambiguous, in our model the mix effect always dominates the volume effect (on platform $B$ ) so that overall consumers benefit from a

\footnotetext{
${ }^{22}$ Since platform $B$ accommodates more firms and its consumer base increases, the total number of relevant ads on platform $B$ increases.
} 
higher targeting ability. The underlying reason for the difference is that in our model the two platforms are competing with each other for consumers, while in Johnson (2013) there is just a single (implicit) platform with exogenously fixed ad price. With competition, each platform has to worry about its consumer base. As a result, when a platform's targeting ability increases, in order to gain more consumer base, it will restrict the increase in the number of participating firms so that the volume effect is smaller than the mix effect.

One may wonder whether the consumer welfare result depends on the asymmetry of the two platforms. The following corollary shows that, with two symmetric platforms, all consumers are unambiguously better off as the targeting ability of both platforms increases.

Proposition 5 Consider two symmetric platforms with $\alpha_{A}=\alpha_{B}=\alpha$. As the targeting ability $\alpha$ increases, each consumer incurs a lower total net nuisance cost.

To summarize, an increase in $\alpha_{B}$ induces both the mix effect and the volume effect on platform $B$, which work in opposite directions. The overall effects on the ad price and the total number of ads on platform $B$ are ambiguous, depending on the distribution of firm types. However, the overall effect on consumer welfare is always positive. The fact that consumers are better off means that the volume effect cannot be relatively too large. But this restriction is not tight enough to ensure that the mix effect dominates the volume effect as to ad price or as to the total number of ads.

On the firms' side, an increase in $\alpha_{B}$ induces some new firms, who initially did not participate on either platform, to participate on platform $B$. Those firms are clearly better off as now they have access to relevant consumers. As to firms who initially participated only on platform $B$, they are also better off with a bigger $\alpha_{B}$, since platform $B$ is now appealing to even lower types. Note that these two groups of firms are the relatively low-profit ones. This result can be considered as one manifestation of the "long tail of advertising": the increase in the targeting ability of the advantaged platform enables less profitable firms, who were previously excluded, to have access to the advertising market and hence consumers. ${ }^{23}$ As to firms participating on both platforms, an increase in $\alpha_{B}$ makes them worse off on platform $A$ (paying a higher ad price), and better off on platform $B$. When the distribution of $v$ is either uniform or exponential, the gain on platform $B$ outweighs the loss on platform $A$, which makes those firms overall better off.

Improve platform $A$ 's targeting ability Now we briefly discuss the case that $\alpha_{A}$ increases to $\alpha_{A}^{\prime} \in\left(\alpha_{A}, \alpha_{B}\right)$, while $\alpha_{B}$ remains the same. It turns out that, compared to the case that $\alpha_{B}$ increases, in most aspects the equilibrium variables of the two individual platforms change

\footnotetext{
${ }^{23}$ See Anderson (2006).
} 
in the reversed directions. In particular, platform $A$ accommodates more firms and platform $B$ reduces the fraction of firms, platform $A$ 's consumer base and profit increase, while those of platform $B$ decrease, platform $B$ 's ad price increases while the change in platform $A$ 's ad price is ambiguous, and the total number of ads on platform $B$ decreases while that on platform $A$ could increase or decrease. However, all consumers are still unambiguously better off from an increase in $\alpha_{A}$. This is because as $\alpha_{A}$ increases, platform $B$ reduces the number of participating firms, which means that consumers on platform $B$ are better off. The fact that platform $A$ gains more consumer base means that consumers on platform $A$ must gain more than those on platform $B$. Combining with previous results, we reach the general conclusion that consumers always benefit from increases in targeting abilities, no matter on which platform the increases occur.

In some aspects, an increase in $\alpha_{A}$ has qualitatively different effects from an increase in $\alpha_{B}$. The first difference concerns the impacts on firms. While an increase in $\alpha_{B}$ benefits low-profit firms, an increase in $\alpha_{A}$ actually makes low-profit firms (initially participating only on platform $B$ ) worse off, since the advantaged platform $(B)$ will accommodate fewer firms and charge a higher ad price. Thus, an increase in the targeting ability of the disadvantaged platform is "anti long tail." The second difference concerns the efficiency of the allocation of consumers. When $\alpha_{B}$ increases, platform $B$ gains consumer base. Since platform $B$ is more efficient than platform $A$, more consumers joining platform $B$ means that the allocation of consumers becomes more efficient. In contrast, when $\alpha_{A}$ increases, the allocation of consumers becomes less efficient as more consumers join the less efficient platform.

\section{$5 \quad$ Investment in Targeting Ability}

In this section, we investigate the platforms' incentives as well as the social planner's incentives to invest in targeting ability. For that purpose, we add an investment stage to the basic model, in which the targeting abilities of platforms are chosen. Specifically, suppose in the investment stage, each platform $i, i=A, B$, simultaneously chooses its own targeting ability $\alpha_{i}$. Two platforms have the same cost function $C\left(\alpha_{i}\right)$, where $C^{\prime}(\cdot) \geq 0$ and $C^{\prime \prime}(\cdot)>0$. We further assume that $C^{\prime}\left(\frac{1}{2}\right)=0$ and $C^{\prime}(\bar{\alpha})=\infty$, where $\bar{\alpha}$ is defined as $\bar{\alpha}=\min \left\{1, \frac{\gamma(1-q)}{(\lambda-\gamma) q+\gamma(1-q)}\right\}{ }^{24}$ These conditions ensure that the resulting targeting abilities for both platforms will be interior: $\alpha_{i} \in(1 / 2, \bar{\alpha})$. At the end of the investment stage, the chosen $\alpha_{A}$ and $\alpha_{B}$ become publicly observable. Then the regular stage of the basic model begins. Note that the two platforms now become symmetric, which can be interpreted as two online platforms competing with each other.

Given $\alpha_{A}$ and $\alpha_{B}$, the equilibrium in the regular stage has been characterized in Section

\footnotetext{
${ }^{24}$ Similar to Condition $1, \alpha<\bar{\alpha}$ ensures that ads overall impose negative externalities on consumers.
} 
3. Denote $\mu_{A}^{*}\left(\alpha_{A}, \alpha_{B}\right)$ and $\mu_{B}^{*}\left(\alpha_{A}, \alpha_{B}\right)$ as the equilibrium fractions of advertising firms. Now consider the choice of targeting ability in the investment stage. We will focus on symmetric equilibrium in which the two platforms choose the same targeting ability. Denote the (symmetric) equilibrium level of targeting ability as $\alpha^{*}$. Suppose platform $j$ chooses the equilibrium targeting ability: $\alpha_{j}=\alpha^{*}$. Then platform $i$ 's profit evaluated at the investment stage is given by

$$
\Pi_{i}\left(\alpha_{i}, \alpha^{*}\right)=\alpha_{i} q \widehat{v}_{i}^{*}\left(\alpha_{i}, \alpha^{*}\right) \mu_{i}^{*}\left(\alpha_{i}, \alpha^{*}\right)\left\{\frac{1}{2}+x\left(\alpha^{*}\right) \mu_{j}^{*}\left(\alpha_{i}, \alpha^{*}\right)-x\left(\alpha_{i}\right) \mu_{i}^{*}\left(\alpha_{i}, \alpha^{*}\right)\right\}-C\left(\alpha_{i}\right) .
$$

Taking the derivative with respect to $\alpha_{i}$, using the Envelope Theorem, and imposing symmetry $\alpha_{i}^{*}=\alpha^{*}$, we get the following first order condition

$$
q \mu^{*} \widehat{v}^{*} \times\left\{\frac{1}{2}+x\left(\alpha^{*}\right) \alpha^{*} \frac{\partial \mu_{j}^{*}\left(\alpha^{*}, \alpha^{*}\right)}{\partial \alpha_{i}}+k \alpha^{*} \mu^{*}\right\}=C^{\prime}\left(\alpha^{*}\right)
$$

where $k=\frac{\partial x}{\partial \alpha}=\frac{1}{2 t}[\lambda q+\gamma(1-2 q)]>0$, and the equilibrium $\mu^{*} \equiv \mu^{*}\left(\alpha^{*}, \alpha^{*}\right)$ is given by

$$
Z^{*} \equiv \frac{\mu^{*} \widehat{v}^{*}}{\widehat{v}^{*}-\frac{1-F\left(\widehat{v}^{*}\right)}{f\left(\widehat{v}^{*}\right)}}=\frac{1}{2 x\left(\alpha^{*}\right)} .
$$

The term $\frac{\partial \mu_{j}^{*}\left(\alpha^{*}, \alpha^{*}\right)}{\partial \alpha_{i}}$ can be calculated as follows:

$$
\begin{aligned}
\frac{\partial \mu_{j}^{*}\left(\alpha^{*}, \alpha^{*}\right)}{\partial \alpha_{i}} & =\frac{k}{x\left(\alpha^{*}\right)} \frac{\frac{d Z^{*}}{d \mu^{*}} \mu^{*}-Z^{*}}{1-\left(1+\frac{d Z^{*}}{d \mu^{*}}\right)^{2}}, \\
\frac{d Z^{*}}{d \mu^{*}} & =\frac{\frac{\left(\mu^{*}\right)^{2}}{\left[f\left(\widehat{v}^{*}\right)\right]^{2}}+\left(\widehat{v}^{*}\right)^{2}+\frac{\left(\mu^{*}\right)^{2} \widehat{v}^{*} f^{\prime}\left(\widehat{v}^{*}\right)}{\left[f\left(\widehat{v}^{*}\right)\right]^{3}}}{\left[\widehat{v}^{*}-\frac{1-F\left(\widehat{v}^{*}\right)}{f\left(\widehat{v}^{*}\right)}\right]^{2}} .
\end{aligned}
$$

From previous results, $\frac{\partial \mu_{j}^{*}\left(\alpha^{*}, \alpha^{*}\right)}{\partial \alpha_{i}}<0$.

An individual platform's incentive to invest in targeting ability, represented by the LHS of (8), can be separated into two effects. The first effect is the profit margin effect, captured by the first term in the braces. An increase in $\alpha$ means a platform can charge a higher price per impression without reducing the number of participating firms. The second effect is the business stealing effect, captured by the last two terms in the braces. An increase in $\alpha_{i}$ would increase platform $i$ 's equilibrium consumer base in the regular stage. While the third term captures the direct impact of changes in $\alpha_{i}$ on the consumer base, the second term is the indirect impact through the changes in platform $j$ 's ad volume.

Now consider the socially optimal level of targeting ability. Suppose a social planner chooses the targeting abilities for both platforms in the investment stage, and then lets the two platforms 
compete by choosing volumes of ads. ${ }^{25}$ The socially optimal solution must be symmetric: the two platforms have the same targeting ability. Given the equilibrium in the later stage $\mu^{*}(\alpha, \alpha)$ (or $\widehat{v}^{*}(\alpha, \alpha)$ ), the social surplus generated by both platforms evaluated at the investment stage is given by

$$
S S(\alpha)=\bar{\beta}-\frac{1}{4} t-y(\alpha) \mu^{*}(\alpha, \alpha)+\alpha q \int_{\widehat{v}^{*}(\alpha, \alpha)}^{\bar{v}} v f(v) d v-2 C(\alpha) .
$$

According to the above expression, the social surplus has five terms. The first term $\bar{\beta}$ is consumers' basic utility of joining platforms. The second term is the total transportation cost incurred by consumers. The third term is the total net nuisance cost suffered by consumers. The fourth term captures the firm surplus generated on two platforms, and the last term is the total investment cost. The socially optimal $\alpha^{o}$ solves

$$
\frac{1}{2}\left[2 t k \mu^{*}-y\left(\alpha^{o}\right) \frac{d \mu^{*}}{d \alpha}\right]+\frac{1}{2} q \int_{\widehat{v}^{*}}^{\bar{v}} v f(v) d v+\frac{1}{2} \alpha^{o} q \widehat{v}^{*} \frac{d \mu^{*}}{d \alpha}=C^{\prime}\left(\alpha^{o}\right),
$$

where

$$
\frac{d \mu^{*}}{d \alpha}=\frac{k}{2\left[x\left(\alpha^{o}\right)\right]^{2} \frac{d Z^{*}}{d \mu^{*}}}, Z^{*}\left(\alpha^{o}\right) \equiv \frac{\mu^{*} \widehat{v}^{*}}{\widehat{v}^{*}-\frac{1-F\left(\widehat{\widehat{*}}^{*}\right)}{f\left(\widehat{v}^{*}\right)}}=\frac{1}{2 x\left(\alpha^{o}\right)} .
$$

The LHS of (10) represents the social incentive to invest in targeting ability. The first term (including the two terms in the brackets) is the consumer surplus effect, which measures how an increase in $\alpha$ impacts consumer surplus. The first term in the brackets is the direct effect: an increase in $\alpha$ reduces consumers' chance of viewing irrelevant ads. The second term in the brackets is an indirect effect: as $\alpha$ increases, both platforms will increase ad volume, which decreases consumer welfare. Overall, the consumer surplus effect is positive (this will be shown later). The second term is the direct effect on firm surplus: an increase in $\alpha$ means that existing firms can be matched with more relevant consumers. The last term is the indirect effect on firm surplus: platforms with a higher $\alpha$ will accommodate more firms, which also contributes to firm surplus. Note that the social incentive to invest does not have a business stealing effect. This is because the social planner internalizes the competition between the two platforms. Moreover, since the platforms are symmetric, there is no distribution effect either.

Now we compare the social incentive and the equilibrium incentive to invest in targeting ability. For that purpose, we assume that $C^{\prime}(\alpha)$ increases fast enough such that both $\alpha^{*}$ and $\alpha^{o}$

\footnotetext{
${ }^{25}$ In an alternative setting, the social planner could choose both the targeting abilities in the first stage and the ad volumes in the second stage. In this setting, the social and equilibrium incentive to invest are further away from each other compared to the setting we are considering.
} 
are unique. Define the LHS of $(8)$ as $H^{*}(\alpha)$ and the LHS of $(10)$ as $H^{o}(\alpha)$. Specifically,

$$
\begin{aligned}
H^{o}(\alpha)-H^{*}(\alpha)= & \frac{1}{2}\left[2 t k \mu^{*}-y(\alpha) \frac{d \mu^{*}}{d \alpha}\right]+\frac{1}{2} q\left[\int_{\widehat{v}^{*}}^{\bar{v}} v f(v) d v-\mu^{*} \widehat{v}^{*}\right] \\
& +\alpha q \widehat{v}^{*}\left[\frac{k}{4 x^{2} \frac{d Z^{*}}{d \mu^{*}}}-k \mu^{*} \frac{Z^{*}+\mu^{*}\left[\frac{d Z^{*}}{d \mu^{*}}+\left(\frac{d Z^{*}}{d \mu^{*}}\right)^{2}\right]}{2 \frac{d Z^{*}}{d \mu^{*}}+\left(\frac{d Z^{*}}{d \mu^{*}}\right)^{2}}\right] .
\end{aligned}
$$

According to (11), given $\alpha$ the difference between the social incentive and equilibrium incentive can be attributed to three terms. The first term is the consumer surplus effect, which is positive and only present under the social incentive. This term implies that in equilibrium platforms tend to underinvest in targeting ability. Intuitively, this is because platforms do not care about consumer surplus per se. The second term is the difference between the direct firm surplus effect under the social incentive and the profit margin effect under the individual incentive. It can be readily seen that this term is positive. Intuitively, as the targeting ability increases, platforms cannot fully appropriate the increase in firm surplus, instead they can only get the increase in the marginal firm's surplus. The third term is the difference between the indirect firm surplus effect under the social incentive and the business stealing effect under the individual incentive. The sign of this term is indeterminate. The following proposition describes situations under which platforms underinvest or overinvest.

Proposition 6 (i) If $q \bar{v} \leq 1 / 2$ and $t \geq 1 / 2$, then $\alpha^{*}<\alpha^{o}$. (ii) Suppose $v$ is uniformly distributed on $[0,1]$. There is an $\widehat{x}$ (defined in the proof) such that if $x(\bar{\alpha}) \geq \widehat{x}$, then $\alpha^{*}<\alpha^{o}$. (iii) Suppose $v$ is uniformly distributed on $[0,1]$. Overinvestment $\left(\alpha^{*}>\alpha^{o}\right)$ occurs only if $t<1 / 2$ and $x(\bar{\alpha})<\widehat{x}$.

To understand part (i) of Proposition 6, note that when $t$ is big the competition between the two platforms is weak. This means that the business stealing effect under the individual incentive is weak. On the other hand, the consumer surplus effect under the social incentive becomes stronger as $t$ increases (the equilibrium $\mu^{*}$ increases and becomes less sensitive to changes in $\alpha$ as the competition between the two platforms becomes weaker). As a result, platforms underinvest in targeting ability in equilibrium if $t$ is big enough.

When firms' profits are uniformly distributed, part (ii) of Proposition 6 provides a condition under which the indirect firm surplus effect under the social incentive dominates the business stealing effect under the individual incentive. Recall that a bigger $x$ implies stronger competition between two platforms. Thus a bigger $x$ implies a lower equilibrium volume of ads, $\mu^{*}$. This tends to reduce the business stealing effect, while the indirect firm surplus effect is more or less the same as the ad volume changes. ${ }^{26}$ Therefore, when $x$ is high enough the indirect

\footnotetext{
${ }^{26}$ In particular, as the ad volume decreases, a given increase in $\alpha_{i}$ will only allow platform $i$ to steal fewer consumers from platform $j$.
} 
firm surplus effect under the social incentive dominates the business stealing effect under the individual incentive, leading to underinvestment in targeting ability in equilibrium.

Overinvestment will occur only if the business stealing effect is stronger than other effects. Part (iii) of Proposition 6, which is directly implied by parts (i) and (ii), specifies the necessary conditions for overinvestment to occur: the transportation cost $t$ should be small, and the nuisance cost of ads $\gamma$ should be relatively small (and the marginal cost of investing in targeting ability is low enough) so that the the equilibrium $\mu^{*}$ is big enough. In the following example overinvestment occurs. Suppose $q=0.1, \gamma=0.001, \lambda=0.001, t=0.00003$, and $C^{\prime}(\alpha)=$ $\max \left\{0, e^{5\left(\frac{20}{19} \alpha-1\right)}-1\right\}$. Then $\alpha^{*}=0.9774>0.9702=\alpha^{o}$. In the numerical examples that we run, it is verified that overinvestment occurs in a very restrictive parameter space, while underinvestment occurs for most of the parameter space. ${ }^{27}$

The result that quantitatively it is hard for overinvestment to occur is somewhat surprising. One would have thought that, as long as the transportation cost $t$ is small enough, the business stealing effect under the individual incentive will be strong enough to dominate and overinvestment will occur. However, as mentioned earlier, if the nuisance cost $\gamma$ does not decrease correspondingly with $t$, stronger competition between two platforms will lead to a small equilibrium $\mu^{*}$ and reduce the profits of platforms. This will dampen platforms' incentive to invest in targeting ability as they will get a lower profit any way.

\section{Conclusion and Discussion}

This paper studies targeted advertising in two-sided markets. Two platforms, with different targeting abilities, compete for single-homing consumers, while advertising firms are multi-homing. Our first set of main results illustrate how asymmetry in targeting ability translates into asymmetric equilibrium outcome, including ad price, total number of ads, consumer welfare, and advertising firms' profits. In particular, our results generate the following potentially testable implications. Compared to the platform with the lower targeting ability, in equilibrium the platform with the higher targeting ability always has more advertising firms, attracts more consumers, and is more profitable. However, the advantaged platform could charge a higher or a lower ad price, and has a higher or lower total number of ads, than the other platform. ${ }^{28}$

Our second set of main results illustrate how an increase in the targeting ability of the advantaged platform affects the equilibrium outcome. First, the disadvantaged platform increases its

\footnotetext{
${ }^{27}$ In a previous version, we also consider an alternative setting in which only platform $B$ chooses targeting ability in the investment stage while platform $A$ has a low and fixed targeting ability (more relevant for an offline platform competing with an online one). In this alternative setting we obtain similar qualitative results.

${ }^{28} \mathrm{In}$ an earlier version of the paper, we also compare the advertising levels and consumer allocation under social optimum with those under equilibrium. Moreover, we also study the situation in which two platforms are owned by a single monopoly, and compare its outcome to the equilibrium outcome under comeptition.
} 
ad price, and its total number of ads decreases; the ad price charged by the advantaged platform and its total number of ads could increase or decrease. Again, these predictions are potentially testable. Second, all consumers, on either platform, are unambiguously better off. Moreover, low-profit advertising firms are better off, and all firms are better off if the profitability of firms follows a uniform or an exponential distribution. When the targeting ability of the disadvantaged platform increases, all consumers are again unambiguously better off, but low-profit firms are worse off.

Finally, we compare social incentives and private incentives to invest in targeting abilities. It turns out that in equilibrium platforms could underinvest as well as overinvest in targeting ability. However, quantitatively underinvestment is much more likely to occur, while overinvestment occurs only under very special conditions.

\section{Appendix}

\section{Proof of Lemma 1.}

Proof. Part (i). Recall that the logconcavity of $f$ implies that $\widehat{v}_{i}-\frac{1-F\left(\widehat{v}_{i}\right)}{f\left(\widehat{v}_{i}\right)}$ is strictly increasing in $\widehat{v}_{i}$. By the definition of $\widetilde{v}$ and $\widetilde{\mu}$, we have $\widehat{v}_{i}-\frac{1-F\left(\widehat{v}_{i}\right)}{f\left(\widehat{v}_{i}\right)} \leq 0$ if $\mu \in[\widetilde{\mu}, 1]$, with the strict inequality if $\mu \neq \widetilde{\mu}$. Since $x_{i} \widehat{v}_{i}\left[1-F\left(\widehat{v}_{i}\right)\right] \geq 0$ for any $\mu$, we reach the conclusion that $\frac{\partial \Pi_{i}}{\partial \mu_{i}}<0$ if $\mu \in(\widetilde{\mu}, 1]$.

Part (ii). A necessary condition for an equilibrium $\mu_{i}^{*}$ is $\frac{\partial \Pi_{i}}{\partial \mu_{i}}\left(\mu_{i}^{*}\right)=0$. Following part (i) we must have $\mu_{i}^{*} \in[0, \widetilde{\mu}]$, if equilibrium exists.

Part (iii). We first show that $\frac{\partial^{2} \Pi_{i}}{\partial \mu_{i}^{2}}<0$ for $\mu_{i} \in[0, \widetilde{\mu})$. By (5),

$$
\frac{\partial^{2} \Pi_{i}}{\partial \mu_{i}^{2}} \propto \frac{\partial\left[\widehat{v}_{i}-\frac{1-F\left(\widehat{v}_{i}\right)}{f\left(\widehat{v}_{i}\right)}\right]}{\partial \mu_{i}} m_{i}\left(\mu_{i}, \mu_{j}\right)+\left[\widehat{v}_{i}-\frac{1-F\left(\widehat{v}_{i}\right)}{f\left(\widehat{v}_{i}\right)}\right] \frac{\partial m_{i}\left(\mu_{i}, \mu_{j}\right)}{\partial \mu_{i}}-x_{i} \frac{\partial\left\{\widehat{v}_{i}\left[1-F\left(\widehat{v}_{i}\right)\right]\right\}}{\partial \mu_{i}} .
$$

Now inspect each term in the above expression. Recall that, for $\mu_{i} \in[0, \widetilde{\mu}), \widehat{v}_{i}-\frac{1-F\left(\widehat{v}_{i}\right)}{f\left(\widehat{v}_{i}\right)}>0$ and is strictly decreasing in $\mu_{i}$. The term $m_{i}\left(\mu_{i}, \mu_{j}\right)$ is also positive and strictly decreasing in $\mu_{i}$. By previous analysis, $\widehat{v}_{i}\left[1-F\left(\widehat{v}_{i}\right)\right]=R\left(\mu_{i}\right)$ is strictly increasing in $\mu_{i}$ for $\mu_{i} \in[0, \widetilde{\mu})$. Therefore, $\frac{\partial^{2} \Pi_{i}}{\partial \mu_{i}^{2}}<0$ for $\mu_{i} \in[0, \widetilde{\mu})$.

Next we show that $\frac{\partial^{2} \Pi_{i}}{\partial \mu_{i} \partial \mu_{j}}>0$. Again by (5),

$$
\frac{\partial^{2} \Pi_{i}}{\partial \mu_{i} \partial \mu_{j}} \propto\left[\widehat{v}_{i}-\frac{1-F\left(\widehat{v}_{i}\right)}{f\left(\widehat{v}_{i}\right)}\right] x_{j},
$$

which is strictly greater than 0 for $\mu_{i} \in[0, \widetilde{\mu})$. 
Claim 1 Let $W(v) \equiv \frac{v}{v-\frac{1-F(v)}{f(v)}}$ and $Z(v) \equiv \frac{v[1-F(v)]}{v-\frac{1-F(v)}{f(v)}}$. Both $W(v)$ and $Z(v)$ are strictly decreasing in $v$ for $v \in[\widetilde{v}, \bar{v})$, and hence are strictly increasing in $\mu$ for $\mu \in[0, \widetilde{\mu})$.

Proof. Taking derivatives, we get

$$
\frac{d W(v)}{d v} \propto-\frac{1-F(v)}{f(v)}+\left(\frac{1-F(v)}{f(v)}\right)^{\prime}<0,
$$

where the inequality uses the fact that $\left(\frac{1-F(v)}{f(v)}\right)^{\prime}<0$ by the logconcavity of $f(\cdot)$. Similarly,

$$
\frac{d Z(v)}{d v} \propto-f(v)\left[v-\frac{1-F(v)}{f(v)}\right]^{2}-v[1-F(v)]\left[v-\frac{1-F(v)}{f(v)}\right]^{\prime}<0,
$$

where the inequality uses the fact that $\left[v-\frac{1-F(v)}{f(v)}\right]^{\prime}$ is strictly increasing in $v$.

\section{Proof of Lemma 2.}

Proof. Recall that, by part (iii) of Lemma $1, \mu_{i}$ and $\mu_{j}$ are strategic complements. And the domain of $[0, \widetilde{\mu}]$ is compact. By Theorem 4.2(ii) of Vives (1990), the set of Nash equilibria is non-empty. To show the uniqueness of Nash equilibrium, we add (6) and (7), and get

$$
\frac{x_{A} \widehat{v}_{A}^{*}\left[1-F\left(\widehat{v}_{A}^{*}\right)\right]}{\widehat{v}_{A}^{*}-\frac{1-F\left(\widehat{v}_{A}^{*}\right)}{f\left(\widehat{v}_{A}^{*}\right)}}+\frac{x_{B} \widehat{v}_{B}^{*}\left[1-F\left(\widehat{v}_{B}^{*}\right)\right]}{\widehat{v}_{B}^{*}-\frac{1-F\left(\widehat{v}_{B}^{*}\right)}{f\left(\widehat{v}_{B}^{*}\right)}}=1 .
$$

Using the definition of $Z(v)$, the above equation can be written compactly as $x_{A} Z\left(\widehat{v}_{A}^{*}\right)+$ $x_{B} Z\left(\widehat{v}_{B}^{*}\right)=1$. Now suppose there are two Nash equilibria: $\left(\mu_{A 1}^{*}, \mu_{B 1}^{*}\right)$ and $\left(\mu_{A 2}^{*}, \mu_{B 2}^{*}\right)$. By Theorem 4.2(ii) Vives (1990), the two Nash equilibria can be ordered. Without loss of generality, suppose $\left(\mu_{A 1}^{*}, \mu_{B 1}^{*}\right)<\left(\mu_{A 2}^{*}, \mu_{B 2}^{*}\right)$. But by the monotonicity of $Z(v)$, according to Claim 1, we have

$$
x_{A} Z\left(\widehat{v}_{A 1}^{*}\right)+x_{B} Z\left(\widehat{v}_{B 1}^{*}\right)<x_{A} Z\left(\widehat{v}_{A 2}^{*}\right)+x_{B} Z\left(\widehat{v}_{B 2}^{*}\right),
$$

which contradicts (12). Therefore, there is a unique equilibrium.

\section{Proof of Proposition 1.}

Proof. Part (i). Suppose, to the contrary, $\mu_{B}^{*} \leq \mu_{A}^{*}$. Since $x_{A}>x_{B}$, we have $x_{A} \mu_{A}^{*}>x_{B} \mu_{B}^{*}$. Taking the difference between (6) and (7), we have

$$
x_{B} \mu_{B}^{*}-x_{A} \mu_{A}^{*}=\frac{1}{2}\left[x_{A} Z\left(\widehat{v}_{A}^{*}\right)-x_{B} Z\left(\widehat{v}_{B}^{*}\right)\right]=\frac{1}{2}\left[x_{A} \mu_{A}^{*} W\left(\widehat{v}_{A}^{*}\right)-x_{B} \mu_{B}^{*} W\left(\widehat{v}_{B}^{*}\right)\right] .
$$

The RHS of the above equation, (13), is strictly greater than 0, which follows from the presumption that $\mu_{B}^{*} \leq \mu_{A}^{*}$, and the facts that $Z(v)>0$ and is increasing in $\mu$ (by Claim 1) for $\mu \in[0, \widetilde{\mu})$, and $x_{A}>x_{B}$. This contradicts the result that the LHS of (13) is strictly less than 0 . Thus we must have $\mu_{B}^{*}>\mu_{A}^{*}$. It immediately follows that $\widehat{v}_{A}^{*}>\widehat{v}_{B}^{*}$. 
To show $x_{A} \mu_{A}^{*}>x_{B} \mu_{B}^{*}$, suppose, to the contrary, $x_{A} \mu_{A}^{*} \leq x_{B} \mu_{B}^{*}$. This means that the LHS of (13) is greater than or equal to 0. Now consider the RHS of (13). By the fact that $\widetilde{\mu}>\mu_{B}^{*}>\mu_{A}^{*}$ and by Claim 1, we have $W\left(\widehat{v}_{B}^{*}\right)>W\left(\widehat{v}_{A}^{*}\right)>0$. Combined with the presumption that $x_{A} \mu_{A}^{*} \leq$ $x_{B} \mu_{B}^{*}$, we draw the conclusion that the RHS of (13) is strictly less than 0 , a contradiction. Therefore, we must have $x_{A} \mu_{A}^{*}>x_{B} \mu_{B}^{*}$. It follows immediately that $m_{A}\left(\mu_{A}^{*}, \mu_{B}^{*}\right)<m_{B}\left(\mu_{A}^{*}, \mu_{B}^{*}\right)$.

The difference in equilibrium profits can be written as

$$
\Pi_{B}^{*}-\Pi_{A}^{*} \propto \alpha_{B} m_{B}^{*} \widehat{v}_{B}^{*}\left[1-F\left(\widehat{v}_{B}^{*}\right)\right]-\alpha_{A} m_{A}^{*} \widehat{v}_{A}^{*}\left[1-F\left(\widehat{v}_{A}^{*}\right)\right] .
$$

Since $\alpha_{B} m_{B}^{*}>\alpha_{A} m_{A}^{*}, \widehat{v}_{B}^{*}\left[1-F\left(\widehat{v}_{B}^{*}\right)\right]-\widehat{v}_{A}^{*}\left[1-F\left(\widehat{v}_{A}^{*}\right)\right] \geq 0$ implies that $\Pi_{B}^{*}-\Pi_{A}^{*}>0$. Recall that $v[1-F(v)]$ is decreasing in $v$ for $v \in[\widetilde{v}, \bar{v}]$. Combining with the result in part (i) that $\widehat{v}_{A}^{*}>\widehat{v}_{B}^{*}$, we have $\widehat{v}_{B}^{*}\left[1-F\left(\widehat{v}_{B}^{*}\right)\right]-\widehat{v}_{A}^{*}\left[1-F\left(\widehat{v}_{A}^{*}\right)\right] \geq 0$. This proves that $\Pi_{A}^{*}<\Pi_{B}^{*}$.

Part (ii). The sign of $p_{B}^{*}-p_{A}^{*}$ can be expressed as

$$
p_{B}^{*}-p_{A}^{*} \propto \frac{\alpha_{B} q \widehat{v}_{B}^{*}}{\alpha_{B} q+\left(1-\alpha_{B}\right)(1-q)}-\frac{\alpha_{A} q \widehat{v}_{A}^{*}}{\alpha_{A} q+\left(1-\alpha_{A}\right)(1-q)} .
$$

By the above expression, $p_{B}^{*}-p_{A}^{*}>0$ is equivalent to

$$
\frac{1+\frac{1-\alpha_{B}}{\alpha_{B}} \frac{1-q}{q}}{1+\frac{1-\alpha_{A}}{\alpha_{A}} \frac{1-q}{q}}<\frac{\widehat{v}_{B}^{*}}{\widehat{v}_{A}^{*}} .
$$

Recall that $\mu \widehat{v}$ is strictly decreasing in $\widehat{v}$ for $\widehat{v} \in[\widetilde{v}, \bar{v})$, which means that $\mu_{B}^{*} \widehat{v}_{B}^{*}>\mu_{A}^{*} \widehat{v}_{A}^{*}$, or $\frac{\mu_{A}^{*}}{\mu_{B}^{*}}<\frac{\widehat{v}_{B}^{*}}{\widehat{v}_{A}^{*}}$. Thus, to prove $p_{A}^{*}<p_{B}^{*}$, it is enough to show that $\frac{1+\frac{1-\alpha_{B}}{\alpha_{B}} \frac{1-q}{q}}{1+\frac{1-\alpha_{A}}{\alpha_{A}} \frac{1-q}{q}} \leq \frac{\mu_{A}^{*}}{\mu_{B}^{*}}$. The fact that $x_{A} \mu_{A}^{*}>x_{B} \mu_{B}^{*}$ implies that $\frac{\mu_{A}^{*}}{\mu_{B}^{*}}>\frac{x_{B}}{x_{A}}$. Thus it is sufficient that $\frac{1+\frac{1-\alpha_{B}}{\alpha_{B}} \frac{1-q}{q}}{1+\frac{1-\alpha_{A}}{\alpha_{A}} \frac{1-q}{q}} \leq \frac{y_{B}}{y_{A}}$.

Part (iii). When both $x_{A}$ and $x_{B}$ goes to 0 , we have $m_{i}^{*} \rightarrow \frac{1}{2}$ and $\mu_{i}^{*} \rightarrow \widetilde{\mu}$, for $i=A, B$. Therefore,

$$
\begin{aligned}
& {\left[\alpha_{A} q+\left(1-\alpha_{A}\right)(1-q)\right] \mu_{A}^{*} m_{A}^{*}-\left[\alpha_{B} q+\left(1-\alpha_{B}\right)(1-q)\right] \mu_{B}^{*} m_{B}^{*} } \\
\propto \quad & {\left[\alpha_{A} q+\left(1-\alpha_{A}\right)(1-q)\right]-\left[\alpha_{B} q+\left(1-\alpha_{B}\right)(1-q)\right]>0, }
\end{aligned}
$$

where the last inequality follows the fact $q<1 / 2$.

\section{Proof of Proposition 2.}

Proof. Part (i). A consumer's total net nuisance cost incurred on platform $i$ is $y_{i} \mu_{i}^{*}$. The fact that $y_{A} \mu_{A}^{*}>y_{B} \mu_{B}^{*}$ follows directly from the result that $x_{A} \mu_{A}^{*}>x_{B} \mu_{B}^{*}$ (part (i) of Proposition 1).

Part (ii). Consider a type $v$ firm advertising on both platforms: $v \geq \widehat{v}_{A}^{*}>\widehat{v}_{B}^{*}$. Its profit per consumer and total profit on platform $i$ are $\alpha_{i} q\left(v-\widehat{v}_{i}^{*}\right)$ and $\alpha_{i} q\left(v-\widehat{v}_{i}^{*}\right) m_{i}^{*}$, respectively. 
Since $\alpha_{A}<\alpha_{B}, \widehat{v}_{A}^{*}>\widehat{v}_{B}^{*}$, and $m_{A}^{*}<m_{B}^{*}$, we conclude that $\alpha_{A} q\left(v-\widehat{v}_{A}^{*}\right)<\alpha_{B} q\left(v-\widehat{v}_{B}^{*}\right)$ and $\alpha_{A} q\left(v-\widehat{v}_{A}^{*}\right) m_{A}^{*}<\alpha_{B} q\left(v-\widehat{v}_{B}^{*}\right) m_{B}^{*}$.

\section{Proof of Proposition 3.}

Proof. Part (i). Since an increase in $\alpha_{B}$ implies a decrease in $x_{B}$, it is sufficient to show that $\frac{\partial \mu_{A}^{*}}{\partial x_{B}}>0$ and $\frac{\partial \mu_{B}^{*}}{\partial x_{B}}<0$. Define $W_{i} \equiv W\left(\widehat{v}_{i}^{*}\right)$. Differentiating (6) and (7) with respect to $x_{B}$, we get

$$
\begin{aligned}
\mu_{B}^{*}+x_{B} \frac{\partial \mu_{B}^{*}}{\partial x_{B}} & =x_{A} \frac{\partial \mu_{A}^{*}}{\partial x_{B}}\left[1+W_{A}+\mu_{A}^{*} \frac{d W_{A}}{d \mu_{A}^{*}}\right], \\
x_{A} \frac{\partial \mu_{A}^{*}}{\partial x_{B}} & =\left(\mu_{B}^{*}+x_{B} \frac{\partial \mu_{B}^{*}}{\partial x_{B}}\right)\left[1+W_{B}\right]+x_{B} \mu_{B}^{*} \frac{d W_{B}}{d \mu_{B}^{*}} \frac{\partial \mu_{B}^{*}}{\partial x_{B}} .
\end{aligned}
$$

From the above two equations, we can solve for $\frac{\partial \mu_{B}^{*}}{\partial x_{B}}$ as follows:

$$
\frac{\partial \mu_{B}^{*}}{\partial x_{B}}=-\frac{\left[\left(1+W_{A}+\mu_{A}^{*} \frac{d W_{A}}{d \mu_{A}^{*}}\right)\left(1+W_{B}\right)-1\right] \mu_{B}^{*}}{\left[\left[1+W_{A}+\mu_{A}^{*} \frac{d W_{A}}{d \mu_{A}^{*}}\right]\left(1+W_{B}+\mu_{B}^{*} \frac{d W_{B}}{d \mu_{B}^{*}}\right)-1\right] x_{B}}<0,
$$

where the inequality follows $\frac{d W_{A}}{d \mu_{A}^{*}}>0$ and $\frac{d W_{B}}{d \mu_{B}^{*}}>0$. From (14) and (16), the sign of $\frac{\partial \mu_{A}^{*}}{\partial x_{B}}$ can be expressed as

$$
\frac{\partial \mu_{A}^{*}}{\partial x_{B}} \propto \mu_{B}^{*}+x_{B} \frac{\partial \mu_{B}^{*}}{\partial x_{B}} \propto\left[1+W_{A}+\mu_{A}^{*} \frac{d W_{A}}{d \mu_{A}^{*}}\right]\left(\mu_{B}^{*} \frac{d W_{B}}{d \mu_{B}^{*}}\right)>0 .
$$

Part (ii). It is sufficient to show that $\frac{\partial\left(x_{A} \mu_{A}^{*}-x_{B} \mu_{B}^{*}\right)}{\partial x_{B}}<0$. More explicitly,

$$
\frac{\partial\left(x_{A} \mu_{A}^{*}-x_{B} \mu_{B}^{*}\right)}{\partial x_{B}}=x_{A} \frac{\partial \mu_{A}^{*}}{\partial x_{B}}-\mu_{B}^{*}-x_{B} \frac{\partial \mu_{B}^{*}}{\partial x_{B}}<0,
$$

where the inequality follows (14), which implies that $\mu_{B}^{*}+x_{B} \frac{\partial \mu_{B}^{*}}{\partial x_{B}}>x_{A} \frac{\partial \mu_{A}^{*}}{\partial x_{B}}$.

Part (iii). Recall that $\widehat{v} \mu>0$ and it is increasing in $\mu$ for $\mu \in[0, \widetilde{\mu}]$. Thus by part (i), we have $\widehat{v}_{A}^{* \prime} \mu_{A}^{* \prime}<\widehat{v}_{A}^{*} \mu_{A}^{*}$ and $\widehat{v}_{B}^{* \prime} \mu_{B}^{* \prime}>\widehat{v}_{B}^{*} \mu_{B}^{*}$. By (4), $\Pi_{i}^{*} \propto \alpha_{i} m_{i}^{*} \widehat{v}_{i}^{*} \mu_{i}^{*}$. Since $\alpha_{A}$ remains the same, $m_{A}^{* \prime}<m_{A}^{*}$ by part (ii), and $\widehat{v}_{A}^{* \prime} \mu_{A}^{* \prime}<\widehat{v}_{A}^{*} \mu_{A}^{*}$, it must be the case that $\Pi_{A}^{* \prime}<\Pi_{A}^{*}$. Similarly, since $\alpha_{B}^{\prime}>\alpha_{B}, m_{B}^{* \prime}>m_{B}^{*}$ by part (ii), and $\widehat{v}_{B}^{* \prime} \mu_{B}^{* \prime}>\widehat{v}_{B}^{*} \mu_{B}^{*}$, we must have $\Pi_{B}^{* \prime}>\Pi_{B}^{*}$.

Part (iv). To show $p_{A}^{* \prime}>p_{A}^{*}$, it is sufficient to show that $\frac{\partial p_{A}^{*}}{\partial x_{B}}<0$. By (1), $\frac{\partial p_{A}^{*}}{\partial x_{B}} \propto \frac{d \widehat{v}_{A}^{*}}{d \mu_{A}^{*}} \frac{\partial \mu_{A}^{*}}{\partial x_{B}}<0$, where the inequality follows $\frac{\partial \mu_{A}^{*}}{\partial x_{B}}>0$ in part (i).

The sign of $p_{B}^{* \prime}-p_{B}^{*}$ can be expressed as

$$
p_{B}^{* \prime}-p_{B}^{*} \propto-\frac{\alpha_{B} q \widehat{v}_{B}^{*}}{\alpha_{B} q+\left(1-\alpha_{B}\right)(1-q)}+\frac{\alpha_{B}^{\prime} q \widehat{v}_{B}^{* \prime}}{\alpha_{B}^{\prime} q+\left(1-\alpha_{B}^{\prime}\right)(1-q)} .
$$


By the above expression, $p_{B}^{* \prime}-p_{B}^{*}>0$ is equivalent to

$$
\frac{1+\frac{1-\alpha_{B}^{\prime}}{\alpha_{B}^{\prime}} \frac{1-q}{q}}{1+\frac{1-\alpha_{B}}{\alpha_{B}} \frac{1-q}{q}}<\frac{\widehat{v}_{B}^{* \prime}}{\widehat{v}_{B}^{*}} .
$$

Since $\widehat{v} \mu>0$ and it is increasing in $\mu$ for $\mu \in[0, \widetilde{\mu}]$, we have $\widehat{v}_{B}^{* \prime} \mu_{B}^{* \prime}-\widehat{v}_{B}^{*} \mu_{B}^{*}>0$, or $\frac{\mu_{B}^{*}}{\mu_{B}^{* \prime}}<\frac{\widehat{v}_{B}^{* \prime}}{\widehat{v}_{B}^{*}}$. Thus to show that $p_{B}^{*}-p_{B}^{* \prime}<0$, it is enough to show that $\frac{1+\frac{1-\alpha_{B}^{\prime}}{\alpha_{B}^{\prime}} \frac{1-q}{q}}{1+\frac{1-\alpha_{B}}{\alpha_{B}} \frac{1-q}{q}} \leq \frac{\mu_{B}^{*}}{\mu_{B}^{* \prime}}$. Note that $x_{B}^{\prime} \mu_{B}^{* \prime}<x_{B} \mu_{B}^{*}$. To see this, by (16) and (17), we have

$$
\frac{\partial\left(x_{B} \mu_{B}^{*}\right)}{\partial x_{B}}=\mu_{B}^{*}+x_{B} \frac{\partial \mu_{B}^{*}}{\partial x_{B}}>0 .
$$

This implies that $\frac{\partial\left(x_{B} \mu_{B}^{*}\right)}{\partial \alpha_{B}}<0$ and $x_{B}^{\prime} \mu_{B}^{* \prime}<x_{B} \mu_{B}^{*}$, since $\mu_{B}^{* \prime}>\mu_{B}^{*}$ by part (i) of Proposition 3. The fact that $x_{B}^{\prime} \mu_{B}^{* \prime}<x_{B} \mu_{B}^{*}$ implies $\frac{y_{B}^{\prime}}{y_{B}}<\frac{\mu_{B}^{*}}{\mu_{B}^{* \prime \prime}}$. Therefore, to show $p_{B}^{*}-p_{B}^{* \prime}<0$ it is sufficient that $\frac{1+\frac{1-\alpha_{B}^{\prime}}{\alpha_{B}^{\prime}} \frac{1-q}{q}}{1+\frac{1-\alpha_{B}}{\alpha_{B}} \frac{1-q}{q}} \leq \frac{y_{B}^{\prime}}{y_{B}}$.

Part (v). Since $\alpha_{A}$ remains the same, $m_{A}^{* \prime}<m_{A}^{*}$, and $\mu_{A}^{* \prime}<\mu_{A}^{*}$, the total number of ads on platform $A$ decreases. Regarding platform $B$, when $t \rightarrow \infty$, we have $m_{B}^{*} \rightarrow \frac{1}{2}, m_{B}^{* \prime} \rightarrow \frac{1}{2}$, $\mu_{B}^{*} \rightarrow \widetilde{\mu}$, and $\mu_{B}^{* \prime} \rightarrow \widetilde{\mu}$. Therefore,

$$
\begin{aligned}
& {\left[\alpha_{B}^{\prime} q+\left(1-\alpha_{B}^{\prime}\right)(1-q)\right] \mu_{B}^{* \prime} m_{B}^{* \prime}-\left[\alpha_{B} q+\left(1-\alpha_{B}\right)(1-q)\right] \mu_{B}^{*} m_{B}^{*} } \\
\propto \quad & {\left[\alpha_{B}^{\prime} q+\left(1-\alpha_{B}^{\prime}\right)(1-q)\right]-\left[\alpha_{B} q+\left(1-\alpha_{B}\right)(1-q)\right]<0, }
\end{aligned}
$$

since $q<1 / 2$. This means that for $t$ big enough, the total number of ads on platform $B$ decreases.

Now we show that the total combined relevant ads increase if the distribution of $v$ is either uniform or exponential. Define $u_{i} \equiv \widehat{v}_{i}-\frac{1-F\left(\widehat{v}_{i}\right)}{f\left(\widehat{v}_{i}\right)}$ and $n_{i} \equiv \widehat{v}_{i} \mu_{i}$. Note that $\frac{\partial n_{i}}{\partial \mu_{i}}=u_{i}>0$, and $\frac{\partial u_{i}}{\partial \mu_{i}}<0$. Now the FOCs of (6) and (7) can be written as:

$$
u_{A}^{*} m_{A}^{*}=x_{A} n_{A}^{*} ; u_{B}^{*} m_{B}^{*}=x_{B} n_{B}^{*} .
$$

Taking derivatives w.r.t. $\alpha_{B}$, we get

$$
\begin{aligned}
\frac{\partial \mu_{B}^{*}}{\partial \alpha_{B}} & =\frac{u_{B}^{*} \frac{\partial m_{B}^{*}}{\partial \alpha_{B}}+k n_{B}^{*}}{x_{B} u_{B}^{*}-\frac{\partial u_{B}^{*}}{\partial \mu_{B}} m_{B}^{*}} \\
\frac{\partial m_{A}^{*}}{\partial \alpha_{B}} & =\frac{x_{A} u_{A}^{*}-\frac{\partial u_{A}^{*}}{\partial \mu_{A}} m_{A}^{*}}{u_{A}^{*}} \frac{\partial \mu_{A}^{*}}{\partial \alpha_{B}}
\end{aligned}
$$

where $k=\frac{1}{2 t}[\lambda q+\gamma(1-2 q)]>0$. The total combined relevant ads are given by $\alpha_{A} q \mu_{A}^{*} m_{A}^{*}+$ $\alpha_{B} q \mu_{B}^{*} m_{B}^{*}$. Taking derivatives w.r.t. $\alpha_{B}$ and using the above equations, we get 


$$
\begin{aligned}
& \frac{\partial\left(\alpha_{A} q \mu_{A}^{*} m_{A}^{*}+\alpha_{B} q \mu_{B}^{*} m_{B}^{*}\right)}{\partial \alpha_{B}} \\
\propto & \alpha_{A} m_{A}^{*} \frac{\partial \mu_{A}^{*}}{\partial \alpha_{B}}+\alpha_{B} m_{B}^{*} \frac{\partial \mu_{B}^{*}}{\partial \alpha_{B}}+\mu_{B}^{*} m_{B}^{*}+\frac{\partial m_{A}^{*}}{\partial \alpha_{B}}\left(\alpha_{A} \mu_{A}^{*}-\alpha_{B} \mu_{B}^{*}\right) \\
> & \left\{\alpha_{A} m_{A}^{*}+\left[\left(\alpha_{A} \mu_{A}^{*}-\alpha_{B} \mu_{B}^{*}\right)-\frac{u_{B}^{*} \alpha_{B} m_{B}^{*}}{x_{B} u_{B}^{*}-\frac{\partial u_{B}^{*}}{\partial \mu_{B}} m_{B}^{*}}\right] \frac{x_{A} u_{A}^{*}-\frac{\partial u_{A}^{*}}{\partial \mu_{A}} m_{A}^{*}}{u_{A}^{*}}\right\} \frac{\partial \mu_{A}^{*}}{\partial \alpha_{B}} .
\end{aligned}
$$

Since $\frac{\partial \mu_{A}^{*}}{\partial \alpha_{B}}<0$, it is enough to show that the term in the braces is negative, which is equivalent to

$$
\begin{aligned}
& \alpha_{A} m_{A}^{*}\left(x_{B} u_{B}^{*}-\frac{\partial u_{B}^{*}}{\partial \mu_{B}} m_{B}^{*}\right) u_{A}^{*}-\alpha_{B} m_{B}^{*}\left(x_{A} u_{A}^{*}-\frac{\partial u_{A}^{*}}{\partial \mu_{A}} m_{A}^{*}\right) u_{B}^{*} \\
& +\left(\alpha_{A} \mu_{A}^{*}-\alpha_{B} \mu_{B}^{*}\right)\left(x_{B} u_{B}^{*}-\frac{\partial u_{B}^{*}}{\partial \mu_{B}} m_{B}^{*}\right)\left(x_{A} u_{A}^{*}-\frac{\partial u_{A}^{*}}{\partial \mu_{A}} m_{A}^{*}\right)<0 .
\end{aligned}
$$

Using the facts that, $\alpha_{A}<\alpha_{B}, x_{A}>x_{B}, m_{A}^{*}<m_{B}^{*}$, and $\frac{\partial u_{i}}{\partial \mu_{i}}<0$, the following condition is sufficient

$$
-\alpha_{A} \frac{\partial u_{B}^{*}}{\partial \mu_{B}} u_{A}^{*}+\alpha_{B} \frac{\partial u_{A}^{*}}{\partial \mu_{A}} u_{B}^{*}+\left(\alpha_{A} \mu_{A}^{*}-\alpha_{B} \mu_{B}^{*}\right) \frac{\partial u_{A}^{*}}{\partial \mu_{A}} \frac{\partial u_{B}^{*}}{\partial \mu_{B}}<0 .
$$

Suppose $v$ is uniformly distributed on $[0,1]$, then $u_{i}^{*}=1-2 \mu_{i}^{*}$, and $\frac{\partial u_{i}^{*}}{\partial \mu_{i}}=-2$. The inequality (18) becomes

$$
\begin{aligned}
& 2 \alpha_{A}\left(1-2 \mu_{A}^{*}\right)-2 \alpha_{B}\left(1-2 \mu_{B}^{*}\right)+4\left(\alpha_{A} \mu_{A}^{*}-\alpha_{B} \mu_{B}^{*}\right) \\
= & 2\left(\alpha_{A}-\alpha_{B}\right)<0,
\end{aligned}
$$

which obviously holds.

Now suppose $v$ has an exponential distribution with density $f(v)=\xi e^{-\xi x}, \xi>0$. In this case, $u_{i}^{*}=\frac{1}{\xi}\left(-\ln \mu_{i}^{*}-1\right)$ and $\frac{\partial u_{i}^{*}}{\partial \mu_{i}}=-\frac{1}{\xi \mu_{i}^{*}}$. The inequality (18) becomes

$$
\begin{aligned}
& -\alpha_{A} \mu_{A}^{*}\left(1+\ln \mu_{A}^{*}\right)+\alpha_{B} \mu_{B}^{*}\left(1+\ln \mu_{B}^{*}\right)+\left(\alpha_{A} \mu_{A}^{*}-\alpha_{B} \mu_{B}^{*}\right) \\
= & \alpha_{B} \mu_{B}^{*} \ln \mu_{B}^{*}-\alpha_{A} \mu_{A}^{*} \ln \mu_{A}^{*}<0 .
\end{aligned}
$$

The above inequality holds because $-\left(\mu_{B}^{*} \ln \mu_{B}^{*}\right)>-\left(\mu_{A}^{*} \ln \mu_{A}^{*}\right)$ by the facts that $-\mu_{i}^{*} \ln \mu_{i}^{*}=$ $\xi n_{i}^{*}, \frac{\partial n_{i}^{*}}{\partial \mu_{i}}>0$, and $\mu_{B}^{*}>\mu_{A}^{*}$.

\section{Proof of Proposition 4.}

Proof. Part (i). Following Proposition 3, we have $\mu_{A}^{* \prime}<\mu_{A}^{*}$. Since $\alpha_{A}$, and hence $y_{A}$, does not change, it implies that $y_{A} \mu_{A}^{* \prime}<y_{A} \mu_{A}^{*}$. The fact that $m_{A}^{* \prime}<m_{A}^{*}$ means that $y_{B}^{\prime} \mu_{B}^{* \prime}-y_{A} \mu_{A}^{* \prime}<$ $y_{B} \mu_{B}^{*}-y_{A} \mu_{A}^{*}$. The above two inequalities imply that $y_{B}^{\prime} \mu_{B}^{* \prime}-y_{B} \mu_{B}^{*}<y_{A} \mu_{A}^{* \prime}-y_{A} \mu_{A}^{*}<0$. 
Part (ii). Firms with $v \in\left(\widehat{v}_{B}^{* \prime}, \widehat{v}_{B}^{*}\right)$ are clearly better off since they were not participating on any platform initially. Firms with $v \in\left[\widehat{v}_{B}^{*}, \widehat{v}_{A}^{*}\right)$ are also better off. To see this, note that before and after the change they only participate on platform $B$. A type $v$ firm's profit on platform $B$ equals to $\alpha_{B} q m_{B}^{*}\left(v-\widehat{v}_{B}^{*}\right)$. Since $\alpha_{B}^{\prime}>\alpha_{B}, m_{B}^{* \prime}>m_{B}^{*}, \widehat{v}_{B}^{* \prime}<\widehat{v}_{B}^{*}$, we have $\alpha_{B}^{\prime} q m_{B}^{* \prime}\left(v-\widehat{v}_{B}^{* \prime}\right)>$ $\alpha_{B} q m_{B}^{*}\left(v-\widehat{v}_{B}^{*}\right)$.

For firms participating on both platforms after the change $\left(v \geq \widehat{v}_{A}^{* \prime}\right)$, a type $v$ firm's total profit on two platforms is given by $\alpha_{A} q m_{A}^{*}\left(v-\widehat{v}_{A}^{*}\right)+\alpha_{B} q m_{B}^{*}\left(v-\widehat{v}_{B}^{*}\right)$. Taking derivatives w.r.t. $\alpha_{B}$, and using similar logic as in the proof of part (iii), in the case of uniform distribution we get

$$
\begin{aligned}
& \frac{\partial\left[\alpha_{A} q m_{A}^{*}\left(v-\widehat{v}_{A}^{*}\right)+\alpha_{B} q m_{B}^{*}\left(v-\widehat{v}_{B}^{*}\right)\right]}{\partial \alpha_{B}}>0 \\
\Leftarrow & -\alpha_{A} \frac{\partial u_{B}^{*}}{\partial \mu_{B}} u_{A}^{*}+\alpha_{B} \frac{\partial u_{A}^{*}}{\partial \mu_{A}} u_{B}^{*}+\left[\alpha_{A}\left(v-\widehat{v}_{A}^{*}\right)-\alpha_{B}\left(v-\widehat{v}_{B}^{*}\right)\right] \frac{\partial u_{A}^{*}}{\partial \mu_{A}} \frac{\partial u_{B}^{*}}{\partial \mu_{B}} \leq 0 \\
\Leftrightarrow & {[2+4(v-1)]\left(\alpha_{A}-\alpha_{B}\right) \leq 0, }
\end{aligned}
$$

which holds, since $v \geq \widehat{v}_{A}^{* \prime} \geq 1 / 2$.

In the case of exponential distribution,

$$
\begin{aligned}
& \frac{\partial\left[\alpha_{A} q m_{A}^{*}\left(v-\widehat{v}_{A}^{*}\right)+\alpha_{B} q m_{B}^{*}\left(v-\widehat{v}_{B}^{*}\right)\right]}{\partial \alpha_{B}}>0 \\
\Leftarrow \quad & 0 \geq \alpha_{A} m_{A}^{*} \frac{u_{A}^{*}}{f\left(\widehat{v}_{A}^{*}\right)}\left(x_{B} u_{B}^{*}-\frac{\partial u_{B}^{*}}{\partial \mu_{B}} m_{B}^{*}\right)-\alpha_{B} m_{B}^{*} \frac{u_{B}^{*}}{f\left(\widehat{v}_{B}^{*}\right)}\left(x_{A} u_{A}^{*}-\frac{\partial u_{A}^{*}}{\partial \mu_{A}} m_{A}^{*}\right) \\
& +\left[\alpha_{A}\left(v-\widehat{v}_{A}^{*}\right)-\alpha_{B}\left(v-\widehat{v}_{B}^{*}\right)\right] \frac{\partial u_{A}^{*}}{\partial \mu_{A}} \frac{\partial u_{B}^{*}}{\partial \mu_{B}}
\end{aligned}
$$

The term $\frac{\alpha_{A} m_{A}^{*} x_{B}}{f\left(\widehat{v}_{A}^{*}\right)}-\frac{\alpha_{B} m_{B}^{*} x_{A}}{f\left(\widehat{v}_{B}^{*}\right)} \propto \frac{\alpha_{A} n_{A}^{*}}{f\left(\widehat{v}_{A}^{*}\right) u_{A}^{*}}-\frac{\alpha_{B} n_{B}^{*}}{f\left(\widehat{v}_{B}^{*}\right) u_{B}^{*}}=\alpha_{A} \frac{1-F\left(\widehat{v}_{A}^{*}\right)}{f\left(\widehat{v}_{A}^{*}\right)} W\left(\widehat{v}_{A}^{*}\right)-\alpha_{B} \frac{1-F\left(\widehat{v}_{B}^{*}\right)}{f\left(\widehat{v}_{B}^{*}\right)} W\left(\widehat{v}_{B}^{*}\right)<0$, since the hazard rate is constant and $W(\cdot)$ is decreasing. Now the original inequality boils down to

$$
\begin{array}{r}
-\alpha_{A} \frac{1-F\left(\widehat{v}_{A}^{*}\right)}{f\left(\widehat{v}_{A}^{*}\right)}\left(1+\ln \mu_{A}^{*}\right)+\alpha_{B} \frac{1-F\left(\widehat{v}_{B}^{*}\right)}{f\left(\widehat{v}_{B}^{*}\right)}\left(1+\ln \mu_{B}^{*}\right)+\alpha_{A}\left(v-\widehat{v}_{A}^{*}\right)-\alpha_{B}\left(v-\widehat{v}_{B}^{*}\right) \leq 0 \\
\Leftarrow-\alpha_{A}\left(1+\ln \mu_{A}^{*}\right)+\alpha_{B}\left(1+\ln \mu_{B}^{*}\right)-\alpha_{B}\left(\ln \mu_{B}^{*}-\ln \mu_{A}^{*}\right) \leq \quad 0 \\
\Leftrightarrow\left(\alpha_{B}-\alpha_{A}\right)\left(1+\ln \mu_{A}^{*}\right) \leq \quad 0,
\end{array}
$$

which obviously holds.

\section{Proof of Proposition 5.}

Proof. Given that $\alpha_{A}=\alpha_{B}=\alpha$, the equilibrium allocation must be symmetric, and we denote the equilibrium variables as $\mu^{*}$ and $\hat{v}^{*}$. By (6) and (7), the equilibrium condition boils down to

$$
y \mu^{*} W^{*}=t
$$


where $y=\gamma(1-q)(1-\alpha)-(\lambda-\gamma) \alpha q$, and $W^{*}=\frac{\hat{v}^{*}}{\hat{v}^{*}-\frac{1-F\left(\hat{w}^{*}\right)}{f\left(\hat{v}^{*}\right)}}$. Taking derivative with respect to $\alpha$ and rearranging, we get

$$
\frac{\partial \mu^{*}}{\partial \alpha}=\frac{-\frac{\partial y}{\partial \alpha} \mu^{*} W^{*}}{y W^{*}+y \mu^{*} \frac{d W^{*}}{d \mu}} .
$$

Recall that a consumer's total net nuisance cost incurred (on either platform) is $y \mu^{*}$. The change in the total net nuisance cost induced by a change in $\alpha$ is given by

$$
\begin{aligned}
\frac{\partial\left(y \mu^{*}\right)}{\partial \alpha} & =\frac{\partial y}{\partial \alpha} \mu^{*}+y \frac{\partial \mu^{*}}{\partial \alpha}=\frac{\partial y}{\partial \alpha} \mu^{*}+y \frac{-\frac{\partial y}{\partial \alpha} \mu^{*} W^{*}}{y W^{*}+y \mu^{*} \frac{d W^{*}}{d \mu}} \\
& =\frac{\partial y}{\partial \alpha} \frac{\mu^{* 2} \frac{d W^{*}}{d \mu}}{W^{*}+\mu^{*} \frac{d W^{*}}{d \mu}}<0,
\end{aligned}
$$

where the inequality uses the facts that $W^{*}>0, \frac{d W}{d \mu}>0$ (Claim 1), and $\frac{\partial y}{\partial \alpha}<0$.

\section{Proof of Proposition 6.}

Proof. Part (i). We first show that the first term in (11) is positive. In particular

$$
\frac{1}{2}\left[2 t k \mu^{*}-y(\alpha) \frac{d \mu^{*}}{d \alpha}\right]=\frac{1}{2} \frac{2 t k}{\frac{d Z^{*}}{d \mu^{*}}}\left(\mu^{*} \frac{d Z^{*}}{d \mu^{*}}-Z^{*}\right) \propto \mu^{*} \frac{d Z^{*}}{d \mu^{*}}-Z^{*},
$$

since $\frac{d Z^{*}}{d \mu^{*}}>0$. But

$$
\mu^{*} \frac{d Z^{*}}{d \mu^{*}}-Z^{*} \propto \frac{\mu^{* 2}}{f^{2}\left(\widehat{v}^{*}\right)}+\frac{\mu^{*} \widehat{v}^{*}}{f^{3}\left(\widehat{v}^{*}\right)}\left[f^{2}\left(\widehat{v}^{*}\right)+f^{\prime}\left(\widehat{v}^{*}\right)\left(1-F\left(\widehat{v}^{*}\right)\right)\right]>0,
$$

where the last inequality uses the fact that the terms in the brackets is positive due to the logconcavity of $f(\cdot)$.

The second term in (11) is clearly positive, since

$$
\int_{\widehat{v}^{*}}^{\bar{v}} v f(v) d v-\mu^{*} \widehat{v}^{*}=\int_{\widehat{v}^{*}}^{\bar{v}}\left(v-\widehat{v}^{*}\right) f(v) d v>0 .
$$

Now we add the first term and the third term of (11) together. Given $q \bar{v} \leq 1 / 2$ and $t \geq 1 / 2$, to show the sum of the two terms is positive, it is sufficient that the following expression is positive:

$$
\left(\mu^{*} \frac{d Z^{*}}{d \mu^{*}}-Z^{*}\right)\left(2+\frac{d Z^{*}}{d \mu^{*}}\right)+\left(Z^{* 2}-\mu^{* 2} \frac{d Z^{*}}{d \mu^{*}}\right)\left(1+\frac{d Z^{*}}{d \mu^{*}}\right)+Z^{*}\left(Z^{*}-\mu^{*}\right)>0 .
$$

Since $Z^{*} \geq \mu^{*}$ and $\mu^{*} \frac{d Z^{*}}{d \mu^{*}}-Z^{*}>0$, the following condition is sufficient:

$$
\left(1+\frac{d Z^{*}}{d \mu^{*}}\right)\left[\mu^{*}\left(1-\mu^{*}\right) \frac{d Z^{*}}{d \mu^{*}}-Z^{*}+Z^{* 2}\right] \geq 0 .
$$


But again by $Z^{*} \geq \mu^{*}$ and $\mu^{*} \frac{d Z^{*}}{d \mu^{*}}-Z^{*}>0$,

$$
\mu^{*}\left(1-\mu^{*}\right) \frac{d Z^{*}}{d \mu^{*}}-Z^{*}+Z^{* 2}>\left(1-\mu^{*}\right) Z^{*}-Z^{*}+Z^{* 2}=Z^{*}\left(Z^{*}-\mu^{*}\right) \geq 0 .
$$

Therefore, when $q \bar{v} \leq 1 / 2$ and $t \geq 1 / 2, H^{o}(\alpha)-H^{*}(\alpha)$ is strictly positive. This further implies that, by the convexity of $C(\cdot), \alpha^{*}<\alpha^{o}$.

Part (ii). Since $v$ is uniformly distributed, $\widetilde{\mu}=1 / 2$, and

$$
Z^{*}=\frac{\mu^{*}\left(1-\mu^{*}\right)}{1-2 \mu^{*}} ; \quad \frac{d Z^{*}}{d \mu^{*}}=1+\frac{2 \mu^{*}\left(1-\mu^{*}\right)}{\left(1-2 \mu^{*}\right)^{2}} .
$$

Now (11) becomes

$$
\begin{aligned}
H^{o}(\alpha)-H^{*}(\alpha) & >\alpha q \widehat{v}^{*}\left[\frac{k}{4 x^{2} \frac{d Z^{*}}{d \mu^{*}}}-k \mu^{*} \frac{Z^{*}+\mu^{*}\left[\frac{d Z^{*}}{d \mu^{*}}+\left(\frac{d Z^{*}}{d \mu^{*}}\right)^{2}\right]}{2 \frac{d Z^{*}}{d \mu^{*}}+\left(\frac{d Z^{*}}{d \mu^{*}}\right)^{2}}\right] \\
& \propto 1-7 \mu^{*}+14 \mu^{* 2}-10 \mu^{* 3}
\end{aligned}
$$

It can be verified that expression (19) $1-7 \mu+14 \mu^{2}-10 \mu^{3}$ is decreasing in $\mu$ for $\mu \in[0,1 / 2]$. Therefore, if $\mu^{*} \leq \widehat{\mu}$, where $\widehat{\mu} \in(0,1 / 2)$ is the solution to $1-7 \mu+14 \mu^{2}-10 \mu^{3}=0$, then $H^{o}(\alpha)-H^{*}(\alpha)>0$. Now define $\widehat{x}$ as $\frac{1}{2 \widehat{x}}=Z(\widehat{\mu})=\frac{\widehat{\mu}(1-\widehat{\mu})}{1-2 \widehat{\mu}}$. We have $\mu^{*} \leq \widehat{\mu}$ if $x \geq \widehat{x}$. Since $x(\alpha)$ reaches its minimum at $\bar{\alpha}, x(\alpha) \geq \widehat{x}$ if $x(\bar{\alpha}) \geq \widehat{x}$. To summarize, if $x(\bar{\alpha}) \geq \widehat{x}$, then $\mu^{*} \leq \widehat{\mu}$, and $H^{o}(\alpha)-H^{*}(\alpha)>0$, which implies $\alpha^{*}<\alpha^{o}$.

Part (iii). Given that $v$ is uniformly distributed, $q \bar{v}<1 / 2$. Thus, by part (i), for $\alpha^{*}>\alpha^{o}$ to occur it must be the case that $t<1 / 2$. By part (ii), for $\alpha^{*}>\alpha^{o}$ to occur it must be the case that $x(\bar{\alpha})<\widehat{x}$.

\section{References}

[1] Ambrus, A., and M. Reisinger. "Exclusive vs Overlapping Viewers in Media Markets." (2006), mimeo.

[2] Anderson, Simon P., and Stephen Coate. "Market Provision of Broadcasting: A Welfare Analysis." The Review of Economic Studies 72, no. 4 (2005): 947-972.

[3] Armstrong, Mark. "Competition in Two-sided Markets." The RAND Journal of Economics 37, no. 3 (2006): 668-691.

[4] Athey, Susan, Emilio Calvano, and Joshua Gans. "The Impact of the Internet on Advertising Markets for News Media." Available at SSRN 2180851 (2012). 
[5] Athey, Susan, and Joshua S. Gans. "The Impact of Targeting Technology on Advertising Markets and Media Competition." American Economic Review, P\&P (2010).

[6] Bergemann, Dirk, and Alessandro Bonatti. "Targeting in Advertising Markets: Implications for Offline versus Online Media." The RAND Journal of Economics 42, no. 3 (2011): 417443.

[7] Bergstrom, Ted, and Mark Bagnoli. "Log-concave Probability and its Applications." Economic Theory 26 (2005): 445-469.

[8] Butters, G. R. "Equilibrium Distribution of Sales and Advertising Prices," Review of Economic Studies, 44(3), 1977, 465-491.

[9] Chandra, Ambarish. "Targeted Advertising: The Role of Subscriber Characteristics in Media Markets." The Journal of Industrial Economics 57, no. 1 (2009): 58-84.

[10] Chandra, Ambarish, and Ulrich Kaiser. "Targeted Advertising in Magazine Markets." No. 10-063. ZEW Discussion Papers, 2010.

[11] de Corniere, Alexandre. "Search Advertising," American Economic Journal: Microeconomics, 8(3), 2016, 156-188.

[12] de Corniere, Alexandre, and Romain de Nijs. "Online Advertising and Privacy," Rand Journal of Economics, 47(1), 2016, 48-72.

[13] Dukes, Anthony. "The Advertsing Market in a Product Oligopoly," Journal of Industrial Economics, 52(3), 2004, 327-348.

[14] Esteban, Lola, Agustin Gil, and Jose M. Hernandez. "Informative Advertising and Optimal Targeting in a Monopoly." The Journal of Industrial Economics 49, no. 2 (2001): 161-180.

[15] Gal-Or, Esther, Mordechai Gal-Or, Jerrold H. May, and William E. Spangler. "Targeted Advertising Strategies on Television." Management Science 52, no. 5 (2006): 713-725.

[16] Galeotti, Andrea, and José Luis Moraga-González. "Segmentation, Advertising and Prices." International Journal of Industrial Organization 26, no. 5 (2008): 1106-1119.

[17] Grossman, G.M., and C. Shapiro. "Informative Advertising with Differentiated Products," Review of Economic Studies, 51(1), 1984, 63-81.

[18] Iyer, Ganesh, David Soberman, and J. Miguel Villas-Boas. "The Targeting of Advertising." Marketing Science 24, no. 3 (2005): 461-476. 
[19] Johnson, Justin P. "Targeted Advertising and Advertising Avoidance." The RAND Journal of Economics 44, no. 1 (2013): 128-144.

[20] Kox, Henk, Bas Straathof, and Gijsbert Zwart. "Targeted Advertising, Platform Competition, and Privacy," Journal of Economics 83 Management Strategy, 26(3), 2017, 557-570.

[21] Masson, Robert, Ram Mudambi, and Robert Reynolds. "Oligopoly in Advertiser-Supported Media," Quarterly Review of Economics and Business, 30(2), 1990, 3-16.

[22] Peitz, Martin, and Tommaso M. Valletti. "Content and Advertising in the Media: Paytv versus Fee-to-air." International Journal of Industrial Organization 26, no. 4 (2008): 949-965.

[23] Rochet, Jean-Charles, and Jean Tirole. "Platform Competition in Two-sided Markets." Journal of the European Economic Association 1, no. 4 (2003): 990-1029.

[24] Rochet, Jean-Charles, and Jean Tirole. "Two-sided Markets: A Progress Report." The RAND Journal of Economics 37, no. 3 (2006): 645-667.

[25] Taylor, Greg. "Attention Retention: Targeted Advertising and the Provision of Media Content." Mimeo, 2011.

[26] Vives, Xavier. "Nash Equilibrium with Strategic Complementarities." Journal of Mathematical Economics 19, no. 3 (1990): 305-321.

[27] Yang, H. "Targeted Search and the Long Tail Effect," Rand Journal of Economics, 44(4), 2013, 733-756.

[28] Zhang, Kaifu, and Zsolt Katona. "Contextual Advertising," Marketing Science, 31(6), 2012, 980-994. 